\title{
Detailed Analysis of the Three Quark Potential in SU(3) Lattice QCD
}

\author{
T.T. Takahashi ${ }^{1}$, H. Suganuma ${ }^{2}$, Y. Nemoto ${ }^{3}$ and H. Matsufuru ${ }^{4}$ \\ ${ }^{1}$ RCNP, Osaka University, Mihogaoka 10-1, Ibaraki, Osaka 567-0047, Japan \\ ${ }^{2}$ Faculty of Science, Tokyo Institute of Technology, Ohokayama 2-12-1, Meguro, Tokyo 152-8551, Japan \\ ${ }^{3}$ Brookhaven National Laboratory, RBRC, Physics Dept. 510A, Upton, New York 11973-5000, USA \\ ${ }^{4}$ YITP, Kyoto University, Kitashirakawa, Sakyo, Kyoto 606-8502, Japan
}

\begin{abstract}
The static three-quark (3Q) potential is studied in detail using SU(3) lattice QCD with $12^{3} \times 24$ at $\beta=5.7$ and $16^{3} \times 32$ at $\beta=5.8,6.0$ at the quenched level. For more than 300 different patterns of the $3 \mathrm{Q}$ systems, we perform the accurate measurement of the $3 \mathrm{Q}$ Wilson loop with the smearing method, which reduces excited-state contaminations, and present the lattice QCD data of the $3 \mathrm{Q}$ ground-state potential $V_{3 \mathrm{Q}}$. We perform the detailed fit analysis on $V_{3 \mathrm{Q}}$ in terms of the Y-ansatz both with the continuum Coulomb potential and with the lattice Coulomb potential, and find that the lattice $\mathrm{QCD}$ data of the $3 \mathrm{Q}$ potential $V_{3 \mathrm{Q}}$ are well reproduced within a few $\%$ deviation by the sum of a constant, the two-body Coulomb term and the three-body linear confinement term $\sigma_{3 \mathrm{Q}} L_{\min }$, with $L_{\min }$ the minimal value of the total length of color flux tubes linking the three quarks. From the comparison with the $\mathrm{Q}-\overline{\mathrm{Q}}$ potential, we find a universality of the string tension as $\sigma_{3 \mathrm{Q}} \simeq \sigma_{\mathrm{Q} \overline{\mathrm{Q}}}$ and the one-gluon-exchange result for the Coulomb coefficients as $A_{3 \mathrm{Q}} \simeq \frac{1}{2} A_{\mathrm{Q} \overline{\mathrm{Q}}}$. We investigate also the several fit analyses with the various ansätze: the Y-ansatz with the Yukawa potential, the $\Delta$-ansatz and a more general ansatz including the $\mathrm{Y}$ and the $\Delta$ ansätze in some limits. All these fit analyses support the Y-ansatz on the confinement part in the $3 \mathrm{Q}$ potential $V_{3 \mathrm{Q}}$, although $V_{3 \mathrm{Q}}$ seems to be approximated by the $\Delta$-ansatz with $\sigma_{\Delta} \simeq 0.53 \sigma$.
\end{abstract}

\section{Introduction}

The strong interaction in hadrons or nuclei is fundamentally ruled by quantum chromodynamics (QCD). In a spirit of the elementary particle physics, it would be desirable to understand hadron physics and nuclear physics at the quark-gluon level based on QCD. However, it still remains as a difficult problem to derive even the inter-quark potential from QCD in the analytic manner, because of the strong-coupling nature of QCD in the infrared region.

In this decade, the lattice QCD calculation has been adopted as a useful and reliable method for the nonperturbative analysis of QCD [1, 2]. In particular, the quark-antiquark (Q- $\bar{Q})$ potential, which is responsible for the meson properties, has been well studied using lattice QCD. The Q- $\bar{Q}$ potential in lattice QCD is well reproduced by a sum of the Coulomb term due to the perturbative one-gluonexchange (OGE) process, and the linear confinement term, besides a irrelevant constant [3, 4, 5. The linear potential at the long distance can be physically interpreted with the flux-tube picture or the string picture for hadrons [6, 7, 8, 9, 10, 11, 12, 13] with the string tension $\sigma \simeq 0.89 \mathrm{GeV} / \mathrm{fm}$, which represents the magnitude of the confinement force. In this picture, the quark and the antiquark are linked with a one-dimensional flux-tube with the string tension $\sigma_{\mathrm{Q}} \overline{\mathrm{Q}}$, and hence the $\mathrm{Q}-\overline{\mathrm{Q}}$ potential is proportional to the distance $r$ between quark and antiquark at the long distance. This flux-tube picture (or the string picture) in the infrared region is supported by the Regge trajectory of hadrons 14, 15], the phenomenological analysis of heavy quarkonia data 16], the strong-coupling expansion of QCD [7] and recent lattice QCD simulations 17, 18, 19].

However, there is almost no reliable formula to describe the three-quark (3Q) potential $V_{3 \mathrm{Q}}$ directly based on QCD, besides the strong-coupling QCD[7, 10], although $V_{3 \mathrm{Q}}$ is directly responsible for the baryon properties 10, 20, 21] and is a primary quantity in the hadron physics. In fact, the 3Q potential has been treated phenomenologically or hypothetically more than 20 years. In contrast with a number of studies on the Q- $\bar{Q}$ potential using lattice QCD [22, 23], there were only a few lattice QCD studies for the $3 \mathrm{Q}$ potential done mainly more than 14 years ago $24,25,26,27$. 
Even at present, the arguments on the $3 \mathrm{Q}$ potential seem to be rather controversial. In Refs. 24, 26, 27, 28, 29, the 3Q potential seemed to be expressed by a sum of two-body potentials, which supports the $\Delta$-type flux tube picture 30]. On the other hand, Ref.[3, 25, 31, 32, 33] seemed to support the Y-type flux-tube picture [10, 20] rather than the $\Delta$-type one. These controversial results may be due to the difficulty of the accurate measurement of the $3 \mathrm{Q}$ ground-state potential in lattice QCD. For instance, in Refs. [24, 26], the authors did not use the smearing for ground-state enhancement, and therefore their results may include serious contamination from the excited-state component. In Refs. 27, 28, 29, the author showed a preliminary result only on the equilateral-triangle case without the fit analysis.

In this paper, for more than 300 different patterns of the $3 \mathrm{Q}$ system, we perform the accurate measurement of the static 3Q potential in $\mathrm{SU}(3)$ lattice $\mathrm{QCD}$ at $\beta=5.7,5.8$ and 6.0 , using the smearing method to remove the excited-state contaminations and to obtain the true ground-state potential. The contents are organized as follows. In Section 2, we make a brief theoretical consideration on the form of the inter-quark potential based on QCD. In Section 3, we explain the method of the lattice QCD measurement of the $3 \mathrm{Q}$ potential, referring the importance of the smearing technique and its physical meaning. In Section 4, we present the lattice QCD data of the 3Q potential, which are accurately measured from the smeared 3Q Wilson loop in a model-independent manner. In Section 5, we perform the fit analysis of the lattice data with the Y-ansatz. In Section 6, we examine the various fit analyses with the $\Delta$-ansatz and a more general ansatz including the $\mathrm{Y}$ and the $\Delta$ ansätze in some limits. Section 7 is devoted to the summary and the concluding remarks.

\section{Theoretical consideration on the $3 \mathrm{Q}$ potential}

The lattice QCD data themselves are measured in a model-independent way, however, it is useful to make a theoretical consideration on the potential form in the static $\mathrm{Q}-\overline{\mathrm{Q}}$ and $3 \mathrm{Q}$ systems with respect to QCD. Although QCD is one of the most difficult theories in the theoretical particle physics, there are two analytical methods based on QCD: one is the perturbative QCD, which is rather established to describe the short-distance behavior, and the other is the strong-coupling expansion, which is expected to reflect the long-distance behavior of QCD. At the short distance, perturbative QCD would work well according to the asymptotic freedom, and the static inter-quark potential can be described with the Coulomb-type potential as the one-gluon-exchange (OGE) result. On the other hand, at the long distance at the quenched level, the flux-tube picture with the string tension $\sigma$ is expected to be applicable from the argument of the strong-coupling expansion of QCD [7, 10, 20], which indicates a linear-type confinement potential proportional to the total flux-tube length. Of course, it is nontrivial that these simple arguments on ultraviolet and infrared limits of QCD hold for the intermediate region as $0.2 \mathrm{fm}<$ $r<1 \mathrm{fm}$. Nevertheless, for instance, the lattice QCD data of the Q- $\bar{Q}$ ground-state potential are well fitted by

$$
V_{\mathrm{Q} \overline{\mathrm{Q}}}(r)=-\frac{A_{\mathrm{Q} \overline{\mathrm{Q}}}}{r}+\sigma_{\mathrm{Q} \overline{\mathrm{Q}}} r+C_{\mathrm{Q} \overline{\mathrm{Q}}}
$$

at the quenched level [5]. Also in the phenomenological aspect of QCD, such a Q- $\bar{Q}$ potential is known to be successful to reproduce the empirical data of the mass spectra and the decay rates of various heavy quarkonia [16. In fact, the Q- $\overline{\mathrm{Q}}$ potential $V_{\mathrm{Q}} \overline{\mathrm{Q}}$ is well described by a sum of the short-distance OGE result and the long-distance flux-tube result.

Also for the $3 \mathrm{Q}$ ground-state potential $V_{3 \mathrm{Q}}$, we basically adopt this picture as a theoretical frame of reference. In the $3 \mathrm{Q}$ system in the color-flux-tube picture, reflecting the character of $\mathrm{SU}\left(N_{c}=3\right)$ in $\mathrm{QCD}$, there can appears the physical junction linking to the three flux tubes stemming from the valence quarks. Since the confinement part is proportional to the total flux-tube length in this picture, the physical junction is expected to appear at the Fermat point of the $3 \mathrm{Q}$ triangle, as shown in Fig. 1, as long as the ground-state $3 \mathrm{Q}$ system with spatially fixed valence quarks is concerned. Here, the Fermat point is defined so as to minimize the sum of the distances to the three vertices of the triangle.

For the convenient description in the argument of the ground-state 3Q potential, we denote by $L_{\text {min }}$ the minimal value of the total length of color flux tubes linking the three quarks. When all angles of 
the $3 \mathrm{Q}$ triangle do not exceed $2 \pi / 3, L_{\min }$ is expressed as

$$
L_{\min }=\left[\frac{1}{2}\left(a^{2}+b^{2}+c^{2}\right)+\frac{\sqrt{3}}{2} \sqrt{(a+b+c)(-a+b+c)(a-b+c)(a+b-c)}\right]^{\frac{1}{2}},
$$

where $a, b$ and $c$ denote the three sides of the $3 \mathrm{Q}$ triangle as shown in Fig. 1. In this case, the physical junction appears and connects the three flux tubes originating from the three quarks, and the shape of the 3Q system is expressed as a Y-type flux tube [7, 10], where the angle between two flux tubes is found to be $2 \pi / 3$ [10, 20]. When an angle of the $3 \mathrm{Q}$ triangle exceeds $2 \pi / 3$, one finds

$$
L_{\min }=a+b+c-\max (a, b, c) .
$$

In the picture of the short-distance OGE result plus the long-distance flux-tube result, the $3 \mathrm{Q}$ ground-state potential $V_{3 \mathrm{Q}}$ is expected to take a form of

$$
V_{3 \mathrm{Q}}=-A_{3 \mathrm{Q}} \sum_{i<j} \frac{1}{\left|\mathbf{r}_{i}-\mathbf{r}_{j}\right|}+\sigma_{3 \mathrm{Q}} L_{\min }+C_{3 \mathrm{Q}}
$$

which is referred to as the Y-ansatz [3, 33, 20]. In the following sections, we will extract first the lattice QCD data of the $3 \mathrm{Q}$ potential without any model assumption, and later we will try the fit analysis of the lattice data with the Y-ansatz or other possible ansätze.

\section{The lattice QCD measurement for the $3 \mathrm{Q}$ potential}

\subsection{The 3Q Wilson loop and the 3Q potential in QCD}

Similar to the derivation of the $\mathrm{Q}-\overline{\mathrm{Q}}$ potential from the Wilson loop, the $3 \mathrm{Q}$ static potential $V_{3 \mathrm{Q}}$ is obtained with the $3 \mathrm{Q}$ Wilson loop as

$$
V_{3 \mathrm{Q}}=-\lim _{T \rightarrow \infty} \frac{1}{T} \ln \left\langle W_{3 \mathrm{Q}}\right\rangle
$$

The $3 \mathrm{Q}$ Wilson loop $W_{3 \mathrm{Q}}$ is defined in a gauge-invariant manner as

$$
W_{3 \mathrm{Q}} \equiv \frac{1}{3 !} \varepsilon_{a b c} \varepsilon_{a^{\prime} b^{\prime} c^{\prime}} U_{1}^{a a^{\prime}} U_{2}^{b b^{\prime}} U_{3}^{c c^{\prime}}
$$

with the path-ordered product

$$
U_{k} \equiv P \exp \left\{i g \int_{\Gamma_{k}} d x_{\mu} A^{\mu}(x)\right\}(k=1,2,3),
$$

along the path denoted by $\Gamma_{k}$ in Fig. 2. As shown in Fig. 2, the 3Q Wilson loop physically expresses the $3 \mathrm{Q}$ gauge-invariant state which is generated at $t=0$ and is annihilated at $t=T$ with the three quarks spatially fixed in $\mathbf{R}^{3}$ for $0<t<T$.

The initial (or the finial) 3Q state is introduced as the string-like object in the naive 3Q Wilson loop. However, the physical ground state of the $3 \mathrm{Q}$ system, which is of interest here, is expected to be expressed by the flux tubes instead of the strings, and then the $3 \mathrm{Q}$ state which is expressed by the strings generally includes excited-state components such as flux-tube vibrational modes. Of course, if the large $T$ limit can be taken, the ground-state potential would be obtained. However, the practical measurement of $\left\langle W_{3 \mathrm{Q}}\right\rangle$ is rather severe for large $T$ in lattice QCD calculations, because $\left\langle W_{3 \mathrm{Q}}\right\rangle$ decreases exponentially with $T$.

Therefore, for the accurate measurement of the $3 \mathrm{Q}$ ground-state potential $V_{3 \mathrm{Q}}$, it is practically indispensable to reduce the excited-state components in the $3 \mathrm{Q}$ system introduced at $t=0$ and $t=T$ in the $3 \mathrm{Q}$ Wilson loop. The gauge-covariant smearing method is one of the most useful technique for the ground-state enhancement [32, 34, 5] without breaking the gauge covariance, and is adopted to measure the $\mathrm{Q}-\overline{\mathrm{Q}}$ potential and the glueball mass 35 in the recent lattice $\mathrm{QCD}$ calculation. (This 
smearing method was not applied to a few pioneering lattice studies on the 3Q potential 24, 26], since the smearing technique was mainly developed after their works. As will be discussed later, their numerical results seem to include fatal large excited-state contaminations.)

In this paper, we perform the accurate measurement of the $3 \mathrm{Q}$ ground-state potential $V_{3 \mathrm{Q}}$, using the ground-state enhancement by the gauge-covariant smearing method for the link-variable in $\mathrm{SU}(3)_{c}$ lattice QCD at the quenched level [32].

\subsection{The smearing method for the ground-state enhancement}

Let us consider here the physical states of the $3 \mathrm{Q}$ system with the spatially fixed quarks. In this $3 \mathrm{Q}$ system, of course, there is no valence-quark motion, and the central issue is the gluonic configuration under the boundary condition of the spatially fixed three quarks, which play the role of the color source of the gluonic color-electric flux.

Like the Q-Q $\mathrm{Q}$ flux-tube system, the ground state of the $3 \mathrm{Q}$ system is expected to be composed by flux tubes rather than the strings 3, 12, and there are many excited states of the 3Q system corresponding to the flux-tube vibrational modes[3]. We here express the 3Q Wilson loop with the normalized physical states, the $3 \mathrm{Q}$ ground state $\mid$ g.s.; $t\rangle$ and the $k$-th excited $3 \mathrm{Q}$ state $\mid k$ th e.s.; $t\rangle$ at $t$. In the $3 \mathrm{Q}$ Wilson loop, the normalized gauge-invariant 3Q state $|3 \mathrm{Q} ; 0\rangle$ created at $t=0$ and $|3 \mathrm{Q} ; T\rangle$ annihilated at $t=T$ can be expressed as

$$
\begin{aligned}
& \left.\left.\left.|3 \mathrm{Q} ; 0\rangle=c_{0} \mid \text { g.s. } ; 0\right\rangle+c_{1} \mid 1 \text { st e.s. } ; 0\right\rangle+c_{2} \mid 2 \text { nd e.s. } ; 0\right\rangle+\ldots, \\
& \left.\left.\left.|3 Q ; T\rangle=c_{0} \mid \text { g.s. } ; T\right\rangle+c_{1} \mid 1 \text { st e.s. } ; T\right\rangle+c_{2} \mid 2 \text { nd e.s. } ; T\right\rangle+\ldots,
\end{aligned}
$$

with the coefficients $c_{i}$ obeying the normalization condition $\sum_{i=0}^{\infty}\left|c_{i}\right|^{2}=1$. Then, the expectation value of $W_{3 \mathrm{Q}}$ can be expressed as

$$
\begin{aligned}
\left\langle W_{3 \mathrm{Q}}(T)\right\rangle & \left.\left.=\langle 3 \mathrm{Q} ; T \mid 3 \mathrm{Q} ; 0\rangle=\left|c_{0}\right|^{2}\langle\text { g.s. } ; T| \text { g.s.; } 0\right\rangle+\left|c_{1}\right|^{2}\langle 1 \text { st e.s. } ; T| 1 \text { st e.s. } ; 0\right\rangle+\ldots \\
& =\left|c_{0}\right|^{2} \exp \left(-V_{\text {g.s. }} T\right)+\left|c_{1}\right|^{2} \exp \left(-V_{1 \text { st e.s. }} T\right)+\ldots
\end{aligned}
$$

with the ground-state potential $V_{\text {g.s. }}$ and the $k$-th excited-state potential $V_{k \text {-th e.s. }}$, which correspond to the energy-eigenvalues of the $3 \mathrm{Q}$ system. (Note that the normalization here is consistent with the definition of $W_{3 \mathrm{Q}}$ in Eq.(6), which leads to $\left\langle W_{3 \mathrm{Q}}(T=0)\right\rangle=1$.)

As increasing $T$, the excited-state components drop faster than the ground-state component in $\left\langle W_{3 \mathrm{Q}}\right\rangle$, however, the ground-state component $\left|c_{0}\right|^{2} \exp \left(-V_{\text {g.s. }} T\right)$ also decreases exponentially. Hence, we face a practical difficulty in extracting the numerical signal. To avoid this difficulty, we adopt the smearing technique 3, 5, 34 which enhances the ground-state overlap as $\left|c_{0}\right|^{2}$ and removes the excitedstate contamination efficiently.

The smearing method is one of the most popular and useful techniques to extract the groundstate potential in lattice QCD. The standard smearing for link-variables is expressed as the iterative replacement of the spatial link-variable $U_{i}(s)(i=1,2,3)$ by the obscured link-variable $\bar{U}_{i}(s) \in \mathrm{SU}(3)_{c}$ [5, 34] which maximizes

$$
\operatorname{Re} \operatorname{tr}\left\{\bar{U}_{i}(s) V_{i}^{\dagger}(s)\right\}
$$

with

$$
V_{i}(s) \equiv \alpha U_{i}(s)+\sum_{j \neq i}\left\{U_{j}(s) U_{i}(s+\hat{j}) U_{j}^{\dagger}(s+\hat{i})+U_{j}^{\dagger}(s-\hat{j}) U_{i}(s-\hat{j}) U_{j}(s+\hat{i}-\hat{j})\right\},
$$

which is schematically illustrated in Fig. 3. Here, $\alpha \in \mathbf{R}$ is referred to as the smearing parameter. The $n$-th smeared link-variables $U_{\mu}^{(n)}(s)\left(n=1,2, . ., N_{\text {smear }}\right)$ are iteratively defined starting from $U_{\mu}^{(0)}(s) \equiv$ $U_{\mu}(s)$ as

$$
U_{i}^{(n)}(s) \equiv \bar{U}_{i}^{(n-1)}(s) \quad(i=1,2,3), \quad U_{4}^{(n)}(s) \equiv U_{4}(s) .
$$

For arbitrary operator $F\left[U_{\mu}(\cdot)\right]$, the $n$-th smeared operator $F\left[U_{\mu}^{(n)}(\cdot)\right]$ is defined with the $n$-th smeared link-variable $U_{\mu}^{(n)}(s)$ instead of the original link-variable. The inter-quark potential can be accurately measured from the properly smeared (3Q) Wilson loop. Here, the smearing parameter $\alpha$ and the iteration number $n$ play the role of the variational parameters and are properly chosen so as to maximize the ground-state component. 
We note that the smearing is just a method to choose the flux-tube-like operator, and hence it never changes the physics itself such as the gauge configuration. As an important feature, this smearing procedure keeps the gauge covariance of the "fat" link-variable $U_{\mu}^{(n)}(s)$ properly. In fact, the gaugetransformation property of $U_{\mu}^{(n)}(s)$ is just the same as that of the original link-variable $U_{\mu}(s)$, and therefore the gauge invariance of $F\left(U_{\mu}^{(n)}(s)\right)$ is ensured for the arbitrary gauge-invariant operator $F\left(U_{\mu}(s)\right)$. For instance, the $n$-th smeared (3Q) Wilson loop is gauge-invariant.

While no temporal extension appears in the smearing, the fat link-variable $U_{\mu}^{(n)}(s)$ includes a spatial extension in terms of the original link-variable $U_{\mu}(s)$, and then the smeared "line" expressed with $U_{\mu}^{(n)}(s)$ physically corresponds to a "flux tube" with the spatial extension. Therefore, if a suitable smearing is done, the smeared line is expected to be close to the ground-state flux tube. This smearing method is actually successful for the extraction of the Q- $\bar{Q}$ potential in lattice QCD [5].

\subsection{The physical meaning of the smearing method}

We consider here the physical meaning of the smearing method with the smearing parameter $\alpha$ in terms of the size or the spatial extension of the $n$-th smeared line. For the convenience of the description, we define

$$
p \equiv \frac{\alpha}{\alpha+4}, \quad q \equiv \frac{1}{\alpha+4},
$$

which satisfy $p+4 q=1$. Let us consider the smearing of the line-like object, which is idealized to be infinitely long. As mentioned above, the smeared line corresponds to the spatially extended flux tube in terms of the original link-variable. Here, we locate the $n$-th smeared line on the $z$-axis in $\mathbf{R}^{3}$, and then, due to the translational invariance along the $z$-direction, the argument is essentially two-dimensional and depends only on $x$ and $y$, and the flux direction is to be in the $z$-direction. We denote by $\varphi(x, y ; n)$ $(x, y \in \mathbf{R})$ the spatial flux distribution in the $n$-th smeared line. (On the lattice with the spacing $a$, $\varphi(x, y ; n)$ is defined on the discrete points $(x, y)=\left(n_{x} a, n_{y} a\right)$ with $n_{x}, n_{y} \in \mathbf{Z}$. $)$

From the iterative definition of the smearing, the spatial flux distribution $\varphi(x, y ; n+1)$ of the $(n+1)$-th smeared line is expected to relate to $\varphi(x, y ; n)$ of the $n$-th smeared line as

$$
\varphi(x, y ; n+1)=p \varphi(x, y ; n)+q\{\varphi(x+a, y ; n)+\varphi(x-a, y ; n)+\varphi(x, y+a ; n)+\varphi(x, y-a ; n)\} .
$$

Here, as shown in Fig. 目 (c), we assume the cancellation of non-z-components of the flux, which exactly holds for the abelian flux. Using the difference operator, we obtain

$$
\begin{aligned}
\Delta_{n} \varphi(x, y ; n) & \equiv \varphi(x, y ; n+1)-\varphi(x, y ; n) \\
& =q\left\{\Delta_{x} \varphi(x, y ; n)-\Delta_{x} \varphi(x-a, y ; n)+\Delta_{y} \varphi(x, y ; n)-\Delta_{y} \varphi(x, y-a ; n)\right\} \\
& =q\left\{\Delta_{x}^{B} \Delta_{x} \varphi(x, y ; n)+\Delta_{y}^{B} \Delta_{y} \varphi(x, y ; n)\right\},
\end{aligned}
$$

where $\Delta_{k}$ and $\Delta_{k}^{B}(k=x, y)$ denote the forward and the backward difference operators satisfying $\Delta_{k} f(\vec{r}) \equiv f(\vec{r}+\hat{k})-f(\vec{r})$ and $\Delta_{k}^{B} f(\vec{r}) \equiv f(\vec{r})-f(\vec{r}-\hat{k})$, respectively.

When the lattice spacing $a$ is small enough, the spatial difference $\Delta_{k}$ can be approximated by the spatial derivative as $\Delta_{k} \simeq a \partial_{k}$. Also for the iteration number $n$ of the smearing, we formally introduce a small "spacing" $a_{n}$ in the " $n$-direction", and define the semi-continuum parameter $\tilde{n} \equiv n a_{n}$, although the final result does not depend on the artificial spacing $a_{n}$. Then, the difference $\Delta_{n}$ can be approximated by the derivative as $\Delta_{n} \simeq a_{n} \partial_{\tilde{n}}$. In this way, we obtain the differential equation as

$$
\frac{\partial}{\partial \tilde{n}} \varphi(x, y ; n)=D\left(\partial_{x}^{2}+\partial_{y}^{2}\right) \varphi(x, y ; n),
$$

which corresponds to the "diffusion equation" at the "time" $\tilde{n}$ with the diffusion parameter

$$
D \equiv \frac{q a^{2}}{a_{n}}=\frac{1}{\alpha+4} \frac{a^{2}}{a_{n}} .
$$

The "initial condition" at $n=0$ is given as

$$
\varphi(x, y ; n=0)=\delta(x) \delta(y),
$$


which means the simple line before applying the smearing. Then, the flux distribution $\varphi(x, y ; n)$ in the $n$-th smeared line can be expressed as

$$
\varphi(x, y ; n)=\frac{1}{(4 \pi D \tilde{n})} \exp \left[-\frac{x^{2}+y^{2}}{4 D \tilde{n}}\right] .
$$

Thus, the $n$-th smeared line physically corresponds to the Gaussian spatially-distributed flux tube in terms of the original link-variable as shown in Fig. 因(d).

As a result, the flux-tube size can be roughly estimated as

$$
R \equiv \sqrt{\left\langle x^{2}+y^{2}\right\rangle} \equiv\left(\frac{\int d x d y \varphi(x, y ; n)\left(x^{2}+y^{2}\right)}{\int d x d y \varphi(x, y ; n)}\right)^{1 / 2}=2 \sqrt{D \tilde{n}}=2 a \sqrt{\frac{n}{\alpha+4}} .
$$

We note that the square root appears as a character of the Brownian motion, and hence $n$-dependence of the flux-tube radius $R$ is not so strong. This formula also explains the physical roles of the two parameters, $\alpha$ and $n$. The smearing parameter $\alpha$ controls the speed of the smearing, and the speed of smearing is slower for larger $\alpha$. For each fixed $\alpha, n$ plays the role of extending the size of the smeared operator. Hence, once we find the suitable smearing parameters $n$ and $\alpha$ which achieve a large ground-state overlap, the physical size of the flux tube is roughly estimated with Eq.(20).

\section{The lattice QCD results of the 3Q potential}

We measure the 3Q potential from the properly smeared 3Q Wilson loop in $\mathrm{SU}(3)_{c}$ lattice QCD at the quenched level. In this section, we present the lattice QCD data of the $3 \mathrm{Q}$ ground-state potential $V_{3 \mathrm{Q}}$ for more than 300 different patterns of the 3Q systems in total. These lattice QCD data are, of course, the model-independent data based on QCD, and we think that the data themselves are useful for the study of the $3 \mathrm{Q}$ system, particularly for the phenomenological approach as the quark model for baryons.

\subsection{The simulation conditions of lattice QCD}

The gauge configurations are generated using the $\mathrm{SU}(3)_{c}$ lattice QCD Monte-Carlo simulation with the standard action with $12^{3} \times 24$ at $\beta=5.7$ and $16^{3} \times 32$ at $\beta=5.8,6.0$ at the quenched level. The pseudo-heat-bath algorithm is adopted for update of the gauge configuration. After a thermalization of more than 5,000 sweeps, we sample the gauge configuration every 500 sweeps, and we use at least 150 gauge configurations at each $\beta$ for the study of the $3 \mathrm{Q}$ potential. We summarize in Table 1 the lattice parameters and the related information on the simulation as well as the lattice spacing $a$ determined so as to reproduce the string tension as $\sigma=0.89 \mathrm{GeV} / \mathrm{fm}$ in the Q- $\overline{\mathrm{Q}}$ potential $V_{\mathrm{Q} \overline{\mathrm{Q}}}$ at each $\beta$. As for the smearing, we set the smearing parameter as $\alpha=2.3$, which is one of the most suitable smearing parameter for the calculation of the $3 \mathrm{Q}$ ground-state potential. The iteration number $N_{\mathrm{smr}}$ of the smearing which maximizes the ground-state overlap and the corresponding flux-tube radius $R$ are also listed at each $\beta$ in Table 1. On the statistical error of the lattice data, we adopt the jackknife error estimate [36]. The Monte-Carlo simulations at $\beta=5.7,6.0$ and 5.8 have been performed on NEC-SX4, NEC-SX5 at Osaka University and HITACHI-SR8000 at KEK, respectively.

\subsection{The $\mathrm{Q}-\overline{\mathrm{Q}}$ potential}

As a frame of reference, we measure the $\mathrm{Q}-\overline{\mathrm{Q}}$ potential $V_{\mathrm{Q}}$ from the properly smeared Wilson loop in the present lattice QCD. As is consistent with the previous lattice works 5], the lattice QCD data of the $\mathrm{Q}-\overline{\mathrm{Q}}$ ground-state potential $V_{\mathrm{Q}} \overline{\mathrm{Q}}$ at the quenched level are well reproduced by

$$
V_{\mathrm{Q} \overline{\mathrm{Q}}}(r)=-\frac{A_{\mathrm{Q} \overline{\mathrm{Q}}}}{r}+\sigma_{\mathrm{Q} \overline{\mathrm{Q}}} r+C_{\mathrm{Q} \overline{\mathrm{Q}}}
$$

with the best-fit parameter set $\left(A_{\mathrm{Q} \overline{\mathrm{Q}}}, \sigma_{\mathrm{Q} \overline{\mathrm{Q}}}, C_{\mathrm{Q} \overline{\mathrm{Q}}}\right)$ listed in Table 2 at each $\beta$. As a visual illustration, we show in Fig. 6 the lattice QCD data of $V_{\mathrm{Q} \overline{\mathrm{Q}}}(r)$ as the function of the inter-quark distance $r$ in the lattice unit. One finds a good agreement of the lattice data of $V_{\mathrm{Q} \bar{Q}}$ and the fit curve with Eq.(21). 
In spite of the visual agreement, the statistical analysis is also necessary for the argument on the fit. This is rather difficult because the lattice QCD data include not only the statistical error but also the systematic errors from the discretization, which cannot be estimated straightforwardly. We here examine the on-axis and the off-axis data at each $\beta$. The on-axis data of $V_{\mathrm{QQ}}$ are well fitted with Eq.(21) at each $\beta$. However, when we include the off-axis data, the fit of $V_{\mathrm{Q} \overline{\mathrm{Q}}}$ with Eq.(21) becomes rather worse as $\chi^{2} / N_{\mathrm{DF}} \sim 10$. This is due to the breaking of the rotational invariance on the lattice, and such breaking is significant for the short-distance data.

As will be discussed in Section 5-5, to be strict, the lattice Coulomb potential would be preferable instead of the Coulomb potential at least for the short-distance lattice data. We examine also the fit with the lattice Coulomb plus linear potential, and list the best-fit parameter set $\left(A_{\mathrm{Q} \bar{Q}}^{\mathrm{LC}}, \sigma_{\mathrm{Q} \overline{\mathrm{Q}}}^{\mathrm{LC}}, C_{\mathrm{Q} \overline{\mathrm{Q}}}^{\mathrm{LC}}\right)$ at each $\beta$ together with $\chi^{2} / N_{\text {DF }}$ in Table 2, where the label as "off-axis" means the fit analysis for both on-axis and off-axis data. We then find that the fit with the lattice Coulomb plus linear potential for both on-axis and off-axis data is fairly good, although the fit parameters such as the string tension are almost unchanged.

\subsection{The ground-state enhancement through the smearing}

Before presenting the lattice data of the $3 \mathrm{Q}$ potential, we briefly demonstrate the utility of the smearing method by estimating the magnitude of the ground-state component in the $3 \mathrm{Q}$ state at $t=0, T$ in the smeared 3Q Wilson loop $W_{3 \mathrm{Q}}$, which is composed with the $n$-th smeared link-variable $U_{\mu}^{(n)}(s)$.

From the similar argument in the $\mathrm{Q}-\overline{\mathrm{Q}}$ system [5], the overlap of the $3 \mathrm{Q}$-state operator with the ground state is estimated with

$$
C_{0} \equiv \frac{\left\langle W_{3 \mathrm{Q}}(T)\right\rangle^{T+1}}{\left\langle W_{3 \mathrm{Q}}(T+1)\right\rangle^{T}}
$$

which is referred to as the ground-state overlap. For instance, in the ideal case where the 3Q state is the perfect ground state in the smeared 3Q Wilson loop, one gets $\left\langle W_{3 \mathrm{Q}}(T)\right\rangle=e^{-V_{3 \mathrm{Q}} T}$ and then $C_{0}=1$. (Here, $W_{3 \mathrm{Q}}(T)$ is normalized as $\left\langle W_{3 \mathrm{Q}}(T=0)\right\rangle=1$, as shown in Eq.(6). In accordance with the excited-state contamination, $C_{0}$ is reduced to be a small value less than unity.

We note that the ground-state potential $V_{3 \mathrm{Q}}$ can be measured accurately if $C_{0}$ is large enough and is close to unity. Then, we check the ground-state overlap $C_{0}$ in the $n$-th smeared 3Q Wilson loop $\left\langle W_{3 \mathrm{Q}}\left(U_{\mu}^{(\cdot)}(s), T\right)\right\rangle$ using lattice QCD simulations, and search reasonable values of the smearing parameter $\alpha$ and the iteration number $N_{\text {smr }}$ of the smearing so as to make $C_{0}$ large. For instance, the ground-state overlap $C_{0}$ is largely enhanced as $0.8<C_{0}<1$ even for $T \leq 3$ by the smearing with $\alpha=2.3$ and $N_{\mathrm{smr}}=12$ for all of the $3 \mathrm{Q}$ configurations at $\beta=5.7$ as shown in Fig. 5 . Thus, the ground-state component is largely enhanced by the suitable smearing.

We list in Table 11 one of the best parameter set $\left(\alpha, N_{\mathrm{smr}}\right)$ at each $\beta$. We note that, as will be shown in the next subsection, the magnitude of the ground-state overlap can be also estimated with $\bar{C}$ in Table 311. One finds a large value of $\bar{C}$ close to unity as $\bar{C} \geq 0.7$ for each lattice data on the 3Q system.

\subsection{The lattice QCD data of the $3 \mathrm{Q}$ potential}

Now, we perform the accurate measurement of the $3 \mathrm{Q}$ ground-state potential $V_{3 \mathrm{Q}}$ using the smearing technique in SU(3) lattice QCD. We investigate more than 300 different patterns of the $3 \mathrm{Q}$ systems in total. In the practical calculation, we consider the following two type $3 \mathrm{Q}$ system on the lattice.

(I) The 3Q system where the three quarks are put on the three spatial axes as $(i, 0,0),(0, j, 0),(0,0, k)$ $(i, j, k=0,1,2, \ldots)$ in $\mathbf{R}^{3}$ in the lattice unit.

(II) The 3Q system where the three quarks are put on the $x$-y plane as $(l, 0,0),(-m, 0,0),(0, n, 0)$ $(l, m, n=0,1,2, \ldots)$ in $\mathbf{R}^{3}$ in the lattice unit.

In both cases, the junction point $\mathrm{O}$ in the $3 \mathrm{Q}$ Wilson loop is set at the origin $(0,0,0)$ in $\mathbf{R}^{3}$, although

the final result of the ground-state potential $V_{3 \mathrm{Q}}$ should not depend on the artificial selection of $\mathrm{O}$. (As will be shown in the fit analysis in Section 5, there is no discontinuity between (I) and (II) on the 3Q 
potential $V_{3 \mathrm{Q}}$, in spite of the fairly different setting of the artificial junction $\mathrm{O}$. This suggests that $V_{3 \mathrm{Q}}$ is independent of $\mathrm{O}$.) For each pattern of the $3 \mathrm{Q}$ system, we calculate the $3 \mathrm{Q}$ Wilson loop of all equivalent $3 \mathrm{Q}$ systems by changing $\mathrm{O}$ and the direction of $\hat{x}, \hat{y}, \hat{z}$, using the translational, the rotational and the reflection symmetries on lattices.

Owing to the smearing, the ground-state component is largely enhanced, and therefore the 3Q Wilson loop $\left\langle W_{3 \mathrm{Q}}\right\rangle$ composed with the smeared link-variable exhibits a single-exponential behavior as

$$
\left\langle W_{3 \mathrm{Q}}\right\rangle \simeq e^{-V_{3 \mathrm{Q}} T}
$$

even for a small value of $T$.

For each $3 \mathrm{Q}$ configuration, we measure $V_{3 \mathrm{Q}}^{\text {latt }}$ from the least squares fit with the single-exponential form

$$
\left\langle W_{3 \mathrm{Q}}\right\rangle=\bar{C} e^{-V_{3 \mathrm{Q}} T} .
$$

Here, we choose the fit range of $T$ such that the stability of the "effective mass"

$$
V(T) \equiv \ln \frac{\left\langle W_{3 \mathrm{Q}}(T)\right\rangle}{\left\langle W_{3 \mathrm{Q}}(T+1)\right\rangle}
$$

is observed to avoid the effect of the excited-state contamination remaining at the small $T$ region. In fact, we use a relatively large value on $T$ as the fit range for the accurate measurement.

In Table 3, we list the lattice QCD data $V_{3 \mathrm{Q}}^{\text {latt }}$ of the $3 \mathrm{Q}$ ground-state potential at $\beta=5.7$, together with the prefactor $\bar{C}$ in Eq.(24), the fit range of $T \in\left[T_{\min }, T_{\max }\right]$ and $\chi^{2} / N_{\mathrm{DF}}$. In Table 4 11, we list up the lattice $\mathrm{QCD}$ data $V_{3 \mathrm{Q}}^{\text {latt }}$ of the $3 \mathrm{Q}$ ground-state potential at $\beta=5.8,6.0$, together with the prefactor $\bar{C}$ in Eq.(24). The statistical error of $V_{3 \mathrm{Q}}^{\text {latt }}$ is estimated with the jackknife method. We stress again that these lattice QCD data are the model-independent data based on QCD, and we think that the data themselves are useful for the study of the 3Q system, particularly for the phenomenological approach as the quark model for baryons.

We note that the prefactor $\bar{C}$ physically means the magnitude of the ground-state overlap in the smeared 3Q Wilson loop. In fact, the pure ground-state $3 \mathrm{Q}$ system leads to $\bar{C}=1$, and $1-\bar{C}$ corresponds to the contribution of the excited-state contamination. We find a large ground-state overlap as $\bar{C} \geq 0.7$ for all $3 \mathrm{Q}$ configurations.

From the best smearing parameters, $\alpha=2.3$ and $N_{\mathrm{smr}}$, the flux-tube radius $R$ can be roughly estimated with Eq. (20) at each $\beta$. We then get a rough estimate of the flux-tube radius as $R \simeq 0.52 \mathrm{fm}$ both at $\beta=5.7,5.8$ and 6.0. This flux-tube radius $R$ seems consistent with the typical hadron size, and it cannot be negligible in comparison with the flux-tube length between the junction and the quark in the $3 \mathrm{Q}$ systems in consideration. In fact, the 3Q systems listed in Table 311 are to be regarded as a flux-tube rather than the string-like object, and hence it is nontrivial whether the strong-coupling QCD can be applicable or not in such $3 Q$ systems. Nevertheless, the Y-ansatz from the simple string picture is found to work well for the lattice QCD data of the three-quark potential.

\section{The fit analysis of the $3 Q$ potential with the Y-ansatz}

For the study of the $3 \mathrm{Q}$ potential $V_{3 \mathrm{Q}}$, we are interested in its large-distance behavior relating to the confinement force rather than the short-distance one. The short-distance behavior of $V_{3 \mathrm{Q}}$ is expected to be described by the two-body Coulomb potential as the one-gluon-exchange (OGE) result in perturbative QCD, although it is nontrivial whether perturbative QCD works well at the intermediate distance as $r \sim 0.5 \mathrm{fm}$. The OGE result indicates also a simple relation on the Coulomb coefficients in the $\mathrm{Q}-\overline{\mathrm{Q}}$ and the $3 \mathrm{Q}$ potentials as $A_{3 \mathrm{Q}} \simeq \frac{1}{2} A_{\mathrm{Q} \overline{\mathrm{Q}}}$.

\subsection{The long-distance behavior of the $3 \mathrm{Q}$ potential}

To begin with, we examine the potential form of $V_{3 \mathrm{Q}}$ at the semi-quantitative level. As the Q-Q potential, the $3 \mathrm{Q}$ potential is also expected to be reproduced by the simple sum of the Coulomb term, the linear confinement term and a constant. In Figs. 74 9, we plot the $3 \mathrm{Q}$ ground-state potential $V_{3 \mathrm{Q}}$ as the function of the minimal total flux-tube length $L_{\min }$, the minimal value of the total length of color 
flux tubes linking the three quarks. as discussed in Section 2. Apart from a constant, $V_{3 \mathrm{Q}}$ is almost proportional to $L_{\min }$ in the infrared region.

To single out the large-distance behavior of $V_{3 \mathrm{Q}}$ by subtracting perturbative Coulomb contribution, we examine $V_{3 \mathrm{Q}}-V_{3 \mathrm{Q}}^{\text {Coul }}$. Here, $V_{3 \mathrm{Q}}^{\text {Coul }}$ is defined as

$$
V_{3 \mathrm{Q}}^{\mathrm{Coul}} \equiv-\frac{A_{\mathrm{Q} \overline{\mathrm{Q}}}}{2} \sum_{i<j} \frac{1}{\left|\mathbf{r}_{i}-\mathbf{r}_{j}\right|},
$$

which is the potential form expected from the OGE process in perturbative QCD. Reflecting the SU(3) color factor, the coefficient in $V_{3 Q}^{\text {Coul }}$ between two quarks, of which combination belong the $\overline{3}$ representation, is set to be a half of the coefficient in the color-singlet $\mathrm{Q}-\overline{\mathrm{Q}}$ system. We note that $A_{\mathrm{Q} \overline{\mathrm{Q}}}$ is already extracted from the lattice QCD data of the Q- $\bar{Q}$ potential, as shown in Table 2 .

In Fig. 10-11, we plot $V_{3 \mathrm{Q}}-V_{3 \mathrm{Q}}^{\text {Coul }}$ as a function of $L_{\min }$, using the lattice data of $V_{3 \mathrm{Q}}$ and $A_{\mathrm{QQ}}$ in Table 2 from the Q- $\bar{Q}$ potential. In the whole region, the linearity on $L_{\min }$ is observed, which means that the $3 \mathrm{Q}$ potential $V_{3 \mathrm{Q}}$ can be well described by a sum of the perturbative Coulomb term as $V_{3 \mathrm{Q}}^{\text {Coul }}$ and the non-perturbative linear confinement term proportional to $L_{\min }$, as shown in Eq.(何). Thus, the lattice data seem to support the Y-ansatz. Note here that this simple fit is not the best fit in terms of the Y-ansatz with $\left(A_{3 \mathrm{Q}}, \sigma_{3 \mathrm{Q}}, C_{3 \mathrm{Q}}\right)$, and the Y-ansatz seems to work well even in this non-best fit. In next subsection, we perform the fit analysis of the $3 \mathrm{Q}$ potential with the Y-ansatz at the quantitative level.

\subsection{The fit analysis with the Y-ansatz}

We perform the best fit analysis for the lattice QCD data of $V_{3 \mathrm{Q}}$ in terms of the Y-ansatz with $\left(A_{3 \mathrm{Q}}, \sigma_{3 \mathrm{Q}}, C_{3 \mathrm{Q}}\right)$ at each $\beta$. We show in Table 2 the best-fit parameter set $\left(A_{3 \mathrm{Q}}, \sigma_{3 \mathrm{Q}}, C_{3 \mathrm{Q}}\right)$ in the Y-ansatz for $V_{3 \mathrm{Q}}$ at each $\beta$. In Table 3 11, we compare the lattice data $V_{3 \mathrm{Q}}^{\text {latt }}$ with the Y-ansatz fitting function $V_{3 \mathrm{Q}}^{\mathrm{fit}}$ in Eq.(四) with the best-fit parameters in Table 2 . We observe a good agreement between $V_{3 \mathrm{Q}}^{\text {latt }}$ and $V_{3 \mathrm{Q}}^{\text {fit }}$. In fact, the deviation $V_{3 \mathrm{Q}}^{\text {latt }}-V_{3 \mathrm{Q}}^{\text {fit }}$ is only within a few $\%$ of the typical scale of $V_{3 \mathrm{Q}}$ for every lattice data in Table 3 11. (Since the potential include an irrelevant constant, the typical scale of $V_{3 \mathrm{Q}}$ is to be understood as its typical variation among the different $3 \mathrm{Q}$ system rather than the value itself.) Thus, the three-quark ground-state potential $V_{3 \mathrm{Q}}$ is well described by Eq.(位) of the Y-ansatz within a few $\%$ deviation.

As a visual demonstration on the agreement of this fit, we compare in Fig. 12 the lattice QCD data $V_{3 \mathrm{Q}}^{\text {latt }}$ at $\beta=5.7$ and the best-fit curve of $V_{3 \mathrm{Q}}^{\text {fit }}$ as the function of $i$ for each $(j, k)$ fixed, when the three quarks are located at $(i, 0,0),(0, j, 0),(0,0, k)$ in the lattice unit. While the lattice data $V_{3 \mathrm{Q}}^{\text {latt }}$ are restricted on the integer of $i$ and are expressed as the points, $V_{3 \mathrm{Q}}^{\mathrm{fit}}$ in Eq.(14) can be calculated for arbitrary real number of $i$ and is expressed as a curve for each $(j, k)$. In Fig. 12 at $\beta=5.7$, one finds a good agreement of the lattice QCD data $V_{3 \mathrm{Q}}^{\text {latt }}$ and the fit curve $V_{3 \mathrm{Q}}^{\mathrm{fit}}$ for each $(j, k)$.

In spite of the good agreement of $V_{3 \mathrm{Q}}^{\text {latt }}$ with $V_{3 \mathrm{Q}}^{\mathrm{fit}}$, to be strict, $\chi^{2} / N_{\mathrm{DF}}$ listed in Table 2 seems relatively large, which means the relatively large deviation $V_{3 \mathrm{Q}}^{\text {latt }}-V_{3 \mathrm{Q}}^{\mathrm{fit}}$ in comparison with the error. Besides physical reasons, this may be due to the under-estimate of the error. In fact, the statistical error itself seems very small, but the error should be inevitably enlarged by the systematic error such as the discretization error in lattice calculations. In particular, the statistical error for the short-distance data is rather small, and such a smallness of the short-distance error seems to provide the large value of $\chi^{2} / N_{\mathrm{DF}}$, which may indicate an importance to control the finite lattice-spacing effect. Of course, this point would be clarified, if the lattice QCD study with the finer and larger lattice is performed. Besides the direct check on the $\beta$-dependence, the similar fit analysis with the lattice Coulomb potential is expected to be meaningful. On the lattice, to be strict, the Coulomb potential is to be modified into the lattice Coulomb potential, which contains the finite lattice-spacing effect more directly. Hence, the fit with the lattice Coulomb potential is expected to reduce the discretization error from the finite lattice spacing, especially for the short-distance data. In the later subsection, we will perform the fit analysis using the lattice Coulomb potential.

Finally, we compare the best-fit parameter set $\left(\sigma_{3 \mathrm{Q}}, A_{3 \mathrm{Q}}, C_{3 \mathrm{Q}}\right)$ in the $3 \mathrm{Q}$ potential $V_{3 \mathrm{Q}}$ in Eq.(牙) with $\left(\sigma_{\mathrm{Q} \overline{\mathrm{Q}}}, A_{\mathrm{Q} \overline{\mathrm{Q}}}, C_{\mathrm{Q} \overline{\mathrm{Q}}}\right)$ in the Q- $\overline{\mathrm{Q}}$ potential $V_{\mathrm{Q} \overline{\mathrm{Q}}}$ in Eq.(21) as listed in Table 2. As a remarkable fact, 
we find a universal feature of the string tension,

$$
\sigma_{3 \mathrm{Q}} \simeq \sigma_{\mathrm{Q} \overline{\mathrm{Q}}}
$$

as well as the OGE result for the Coulomb coefficient,

$$
A_{3 \mathrm{Q}} \simeq \frac{1}{2} A_{\mathrm{Q} \overline{\mathrm{Q}}}
$$

\subsection{The model-independent check in the diquark limit}

As a model-independent check, we consider the diquark limit, where two quark locations coincide in the $3 \mathrm{Q}$ system. In the diquark limit, the static $3 \mathrm{Q}$ system becomes equivalent to the $\mathrm{Q}-\overline{\mathrm{Q}}$ system, which leads to a physical requirement on the relation between $V_{3 \mathrm{Q}}$ and $V_{\mathrm{Q} \overline{\mathrm{Q}}}$. Our results, $\sigma_{3 \mathrm{Q}} \simeq \sigma_{\mathrm{Q} \overline{\mathrm{Q}}}$ and $A_{3 \mathrm{Q}} \simeq \frac{1}{2} A_{\mathrm{Q} \overline{\mathrm{Q}}}$, are consistent with the physical requirement in the diquark limit.

Next, we consider the constant terms $C_{3 \mathrm{Q}}$ in the diquark limit, although such a constant term is a lattice artifact and is physically irrelevant. As a caution in the continuum diquark limit, there appears a singularity or a divergence from the Coulomb term in $V_{3 \mathrm{Q}}$ as

$$
\lim _{\mathbf{r}_{j} \rightarrow \mathbf{r}_{i}} \frac{-A_{3 \mathrm{Q}}}{\left|\mathbf{r}_{i}-\mathbf{r}_{j}\right|}=-\infty
$$

In the lattice regularization, this ultraviolet divergence is regularized to be a finite constant with the lattice spacing $a$ as

$$
\frac{-A_{3 \mathrm{Q}}}{\left|\mathbf{r}_{i}-\mathbf{r}_{j}\right|} \rightarrow \frac{-A_{3 \mathrm{Q}}}{\omega a}
$$

where $\omega$ is a dimensionless constant satisfying $0<\omega<1$ and $\omega \sim 1$. Then, we find

$$
C_{3 \mathrm{Q}}+\frac{-A_{3 \mathrm{Q}}}{\omega a}=C_{\mathrm{Q} \overline{\mathrm{Q}}}
$$

or equivalently,

$$
C_{3 \mathrm{Q}}-C_{\mathrm{Q} \overline{\mathrm{Q}}}=\frac{A_{3 \mathrm{Q}}}{\omega a} \quad(>0)
$$

in the diquark limit. This is the requirement for the constant term in the diquark limit on the lattice. Our lattice QCD results for $C_{3 \mathrm{Q}}, C_{\mathrm{QQ}}$ and $A_{3 \mathrm{Q}}$ are thus consistent with this requirement, and one finds $\omega \simeq 0.41-0.45: \omega(\beta=5.7) \simeq 0.447, \omega(\beta=5.8) \simeq 0.414, \omega(\beta=6.0) \simeq 0.424$.

\subsection{The Y-ansatz with the Yukawa potential}

In the previous subsection, we adopt the Coulomb potential as the short-distance ingredient, because the OGE process is expected to be dominant at the short distance and the $\mathrm{Q}-\overline{\mathrm{Q}}$ potential seems to be reproduced with the Coulomb plus linear potential in the lattice QCD. The first reason is, however, nontrivial in the intermediate and the infrared regions, where the perturbative QCD would not work. In fact, due to some nonperturbative effects besides the confinement potential, the Coulomb potential caused by the OGE process may be modified in the infrared region.

For instance, the dual superconductor theory for the quark confinement [12, 13, 37, 38] supports the Yukawa plus linear potential rather than the Coulomb plus linear potential, although the dual gluon mass $m_{B}$ appearing in the exponent in the Yukawa potential may not be so large, e.g., $m_{B} \sim 0.5 \mathrm{GeV}$ both in the model framework [12, 13] and in the lattice study [39, 40].

From the theoretical viewpoint, such a possibility on the infrared screening of the Coulomb potential seems rather attractive in terms of the empirical absence of the color Van-Der-Waals force in the infrared limit 41. In fact, if the two-body Coulomb potential is not screened in the infrared limit, the color VanDer-Waals force inevitably appears as a long-distance force between hadrons, which is not observed experimentally.

Then, we also investigate the fit analysis of $V_{3 \mathrm{Q}}$ with the Y-ansatz with the Yukawa potential as

$$
V_{3 \mathrm{Q}}^{\text {Yukawa }} \equiv-A_{3 \mathrm{Q}}^{\text {Yukawa }} \sum_{i<j} V^{\text {Yukawa }}\left(\left|\mathbf{r}_{i}-\mathbf{r}_{j}\right|\right)+\sigma_{3 \mathrm{Q}}^{\text {Yukawa }} L_{\min }+C_{3 \mathrm{Q}}^{\text {Yukawa }},
$$


where $V^{\text {Yukawa }}(r)$ denotes the normalized Yukawa potential

$$
V^{\text {Yukawa }}(r) \equiv \frac{1}{r} e^{-m_{B} r}
$$

Here, $m_{B}$ corresponds to the dual gluon mass in the dual superconductor picture [6, 12, 13].

We find that the best-fit analysis of the lattice QCD data $V_{3 \mathrm{Q}}^{\text {latt }}$ with $V_{3 \mathrm{Q}}^{\text {Yuka }}$ indicates $m_{B} \simeq 0$. Of course, in this special case of $m_{B} \simeq 0$, the Yukawa potential reduces the Coulomb potential, and the result almost coincides with that in the previous fit. Thus, through the fit analysis with the Yukawa potential based on the Y-ansatz, we have observed no definite evidence to replace the Coulomb-potential part by the Yukawa potential in the present calculation.

\subsection{The Y-ansatz with the lattice Coulomb potential}

So far, we have investigated the fit analysis of the $3 \mathrm{Q}$ potential $V_{3 \mathrm{Q}}$ mainly with the continuum Coulomb and the confinement potential. However, in comparing with the lattice data, the careful treatment considering the lattice discretization effect may be desired. The main effect of the lattice discretization appears only at the short distance, and hence no modification would be necessary for the confinement potential, which becomes significant only at the large distance. On the other hand, it is nontrivial to use the continuum Coulomb potential for the fit of the lattice data, especially at the short distance, according to the lattice discretization. For instance, the short-distance singularity of the Coulomb potential becomes rather smeared on the lattice, as will be shown later.

In this subsection, we perform the fit analysis of the lattice QCD data of $V_{3 \mathrm{Q}}$ in terms of the Y-ansatz with the lattice Coulomb potential. The lattice Coulomb (LC) potential between two color charges is obtained with the lattice Coulomb propagator as

$$
V^{\mathrm{LC}}(\vec{n}) \equiv \pi \int_{-\pi / a}^{\pi / a} \frac{d^{3} q}{(2 \pi)^{3}} \frac{\exp (-i \vec{p} \cdot \vec{n} a)}{\sum_{i=1}^{3} \sin ^{2}\left(p_{i} a / 2\right)}
$$

where $\vec{n} \equiv\left(n_{1}, n_{2}, n_{3}\right) \in \mathbf{Z}^{3}$ denotes the relative vector between the two color charges in the lattice unit. Here, the lattice Coulomb potential $V^{\mathrm{LC}}(\vec{n})$ is properly normalized so as to reduce into the $1 / \mathrm{r}$ potential in the continuum limit as

$$
V^{\mathrm{LC}}(\vec{n}) \rightarrow \frac{1}{r}
$$

with $r=|\vec{n}| a$.

In Fig. 13, we plot $V^{\mathrm{LC}}(\vec{n})$ as a function of $|\vec{n}|$ together with $1 / r$ as a function of $r$. One find that the singularity near the origin $|\vec{n}|=0$ becomes smeared, and $V_{\mathrm{LC}}$ takes a finite value even at $\vec{n}=\overrightarrow{0}$, which was mentioned in the context of the diquark limit in the previous subsection. This may cause a significant deviation in the fit analysis with the $1 / r$ Coulomb potential, especially for the short-distance data analysis.

To begin with, we examine the fit analysis of the lattice $\mathrm{QCD}$ data on the Q- $\overline{\mathrm{Q}}$ potential $V_{\mathrm{Q} \overline{\mathrm{Q}}}$ with

$$
V_{\mathrm{Q} \overline{\mathrm{Q}}}^{L C} \equiv-A_{\mathrm{Q} \overline{\mathrm{Q}}}^{\mathrm{LC}} V^{\mathrm{LC}}(\vec{n})+\sigma_{\mathrm{Q} \overline{\mathrm{Q}}}^{\mathrm{LC}}|\vec{n}|+C_{\mathrm{Q} \overline{\mathrm{Q}}}^{\mathrm{LC}},
$$

using the lattice Coulomb potential $V^{\mathrm{LC}}(\vec{n})$. We refer to this fit as the LC fit. We list in Table 2 the best-fit parameter set $\left(A_{\mathrm{Q} \bar{Q}}^{\mathrm{LC}}, \sigma_{\mathrm{Q} \bar{Q}}^{\mathrm{LC}}, C_{\mathrm{Q} \overline{\mathrm{Q}}}^{\mathrm{LC}}\right)$ at each $\beta$. In Table 2, the label "on-axis" means the fit analysis for the on-axis data only, and the label "off-axis" means the fit analysis for both on-axis and off-axis data. In the LC fit, a significant reduction of $\chi^{2} / N_{\mathrm{DF}}$ is observed, in comparison with the fit with the 1/r Coulomb potential in Eq.(21), as shown in Table 2, in spite of the similar values of the fit parameters. We find a further good agreement between the lattice QCD data and the fitted values of $V_{\mathrm{Q} \overline{\mathrm{Q}}}^{\mathrm{LC}}$ on the Q- $\overline{\mathrm{Q}}$ potential. In particular, the LC fit shows an acceptable value of $\chi^{2} / N_{\mathrm{DF}}$ even for the fit on both on-axis and off-axis data, while the fit with the $1 / r$ Coulomb potential shows the extremely large $\chi^{2} / N_{\mathrm{DF}}$ about 10 when the off-axis data are included. Thus, the discretization effect, which may not be negligible for short-distance data, seems to be taken into account neatly by the use of the LC potential to some extent. Accordingly, the fit analysis with the lattice Coulomb potential $V^{\mathrm{LC}}$ seems to 
provide a more precise information also for the linear confinement potential, although the string tension obtained from the fit analysis is almost unchanged as $\sigma_{\mathrm{Q} \overline{\mathrm{Q}}}^{\mathrm{LC}} \simeq \sigma_{\mathrm{Q} \overline{\mathrm{Q}}}$.

Now, we perform the LC fit analysis of the lattice data on the $3 \mathrm{Q}$ potential $V_{3 \mathrm{Q}}$ with

$$
V_{3 \mathrm{Q}}^{\mathrm{LC}} \equiv-A_{3 \mathrm{Q}}^{\mathrm{LC}} \sum_{i<j} V^{\mathrm{LC}}\left(\overrightarrow{n_{i j}}\right)+\sigma_{3 \mathrm{Q}}^{\mathrm{LC}} L_{\min }+C_{3 \mathrm{Q}}^{\mathrm{LC}}
$$

using the lattice Coulomb potential $V^{\mathrm{LC}}(\vec{n})$. In Table 2, we list the best-fit parameter set $\left(A_{3 \mathrm{Q}}^{\mathrm{LC}}\right.$, $\left.\sigma_{3 \mathrm{Q}}^{\mathrm{LC}}, C_{3 \mathrm{Q}}^{\mathrm{LC}}\right)$ together with the best-fit parameters $\left(A_{\mathrm{Q} \overline{\mathrm{Q}}}^{\mathrm{LC}}, \sigma_{\mathrm{Q} \overline{\mathrm{Q}}}^{\mathrm{LC}}, C_{\mathrm{Q} \overline{\mathrm{Q}}}^{\mathrm{LC}}\right)$. In this $\mathrm{LC}$ fit, $\chi^{2} / N_{\mathrm{DF}}$ is reduced in comparison with the fit with the $1 / r$ Coulomb potential at each $\beta$, as shown in Table 2. Then, this fit seems to be acceptably good even without taking account of the remaining systematic error. Accordingly, the 3Q potential data are well reproduced with the Y-ansatz fit function $V_{3 \mathrm{Q}}^{\mathrm{LC}}$ in Eq.(38) with the lattice Coulomb potential with accuracy better than $1 \%$. Again, the fit analysis with the lattice Coulomb potential $V^{\mathrm{LC}}$ is expected to control the discretization effect to some extent, and would provide a more precise information also for the linear confinement potential.

Finally, we focus on the best-fit parameter set $\left(\sigma_{3 \mathrm{Q}}^{\mathrm{LC}}, A_{3 \mathrm{Q}}^{\mathrm{LC}}, C_{3 \mathrm{Q}}^{\mathrm{LC}}\right)$ in the Y-ansatz with the lattice Coulomb potential. The values of the best-fit parameters in the LC fit are almost the same as those in the previous fit with the $1 / r$ Coulomb potential as

$$
\left(\sigma_{3 \mathrm{Q}}^{\mathrm{LC}}, A_{3 \mathrm{Q}}^{\mathrm{LC}}, C_{3 \mathrm{Q}}^{\mathrm{LC}}\right) \simeq\left(\sigma_{3 \mathrm{Q}}, A_{3 \mathrm{Q}}, C_{3 \mathrm{Q}}\right) .
$$

In particular, the string tension obtained from the fit analysis is almost unchanged as $\sigma_{3 \mathrm{Q}}^{\mathrm{LC}} \simeq \sigma_{3 \mathrm{Q}}$. We then find again the universality of the string tension as $\sigma_{3 \mathrm{Q}}^{\mathrm{LC}} \simeq \sigma_{\mathrm{Q} \overline{\mathrm{Q}}}^{\mathrm{LC}}$ at each $\beta$. The OGE relation on the Coulomb coefficient is found as $A_{3 \mathrm{Q}}^{\mathrm{LC}} \simeq \frac{1}{2} A_{\mathrm{Q} \overline{\mathrm{Q}}}^{\mathrm{LC}}$. In particular, this OGE relation seems to be observed precisely at $\beta=6.0$, which is the finest and the most reliable lattice in the present calculation. (At $\beta=5.7$, 5.8 , the ratio $A_{3 \mathrm{Q}}^{\mathrm{LC}} / A_{\mathrm{Q} \overline{\mathrm{Q}}}^{\mathrm{LC}}$ is about 0.4 , which seems slightly different from $\frac{1}{2}$ as the OGE result. Note that, however, the discretization error on the QCD action still remains at the small $\beta$ even with the lattice Coulomb potential. In addition, at $\beta=5.7,5.8$, the nearest site is relatively far, and hence the Coulomb contribution to the $3 \mathrm{Q}$ potential is relatively small, which may lead to an uncertainty of the Coulomb coefficient.)

To summarize this section, we conclude that the lattice $\mathrm{QCD}$ data of the $3 \mathrm{Q}$ potential $V_{3 \mathrm{Q}}$ can be fairly described with the $\mathrm{Y}$-ansatz within a few $\%$ deviation, and therefore the nonperturbative linear confinement potential is proportional to $L_{\min }$, the minimal value of the total length of the Y-type flux tube linking the three quarks, which supports the Y-ansatz.

\section{Comparison with the delta ansatz and the generalized Y- ansatz}

\subsection{Comparison with the Delta ansatz}

For the $3 \mathrm{Q}$ potential, the $\Delta$-ansatz is also an interesting candidate [30] as well as the Y-ansatz. The $\Delta$-ansatz is expressed as

$$
V_{3 \mathrm{Q}}=-A_{\Delta} \sum_{i<j} \frac{1}{\left|\mathbf{r}_{i}-\mathbf{r}_{j}\right|}+\sigma_{\Delta} \sum_{i<j}\left|\mathbf{r}_{i}-\mathbf{r}_{j}\right|+C_{\Delta},
$$

which consists of the two-body linear potential between quarks. This $\Delta$-ansatz has been adopted in a simple nonrelativistic quark potential model [42], because of its simplicity for the calculation. In addition, several lattice QCD studies for the 3Q potential supported the $\Delta$ ansatz [24, 26, 27, 28, 29]. However, Refs. 24, 26] seem rather old done in 14 years ago, and were done without smearing, so that the excited-state potential may largely contribute in their measurements. (See Fig. 5.) In recent paper as Ref. [29], in spite of the use of the smearing, the authors simply compared the 3Q potential $V_{3 \mathrm{Q}}$ with the $\mathrm{Y}$ and the $\Delta$ ansätze with a fixed string tension estimated from the Q- $\overline{\mathrm{Q}}$ potential, only for several equilateral-triangle $3 \mathrm{Q}$ configurations without the quantitative fit analysis. Furthermore, 
in Refs. 27, 29], the "deviation" between the lattice data and the Y-ansatz seems to be explained as a trivial constant shift of the potential. In fact, the potential calculated in lattice QCD includes a physically irrelevant constant, which is not properly scaled in the physical unit, as shown in Table 2. If such an irrelevant constant is properly removed, the Y-ansatz seems to be better than $\Delta$-ansatz even in Refs. [27, 28, 29], e.g., the slope of the lattice data $V_{3 \mathrm{Q}}^{\text {latt }}$ seems closer to the Y-ansatz rather than the $\Delta$-ansatz. In Ref. [29], the authors set the potential origin at the nearest lattice point and used the continuum Coulomb potential for the fit function, but this seems dangerous because a constant deviation may appear as a mismatch between the lattice Coulomb potential which is preferable to the lattice data and the continuum Coulomb potential for the fit function, as shown in Fig. 13. In any case, the quantitative fit analysis is essential for the study of the functional form of the $3 \mathrm{Q}$ potential.

In this subsection, we perform the fit analysis with the $\Delta$ ansatz. To begin with, we try to fit $V_{3 \mathrm{Q}}^{\text {latt }}$ with the $\Delta$-ansatz in Eq.(40), which was suggested in Refs. 24, 26, 30. We list in Table 12 the best fit parameter set $\left(A_{\Delta}, \sigma_{\Delta}, C_{\Delta}\right)$ in the $\Delta$-ansatz at each $\beta$. In comparison with the Y-ansatz, this fit with the $\Delta$-ansatz seems rather worse, because of the larger value of $\chi^{2} / N_{\mathrm{DF}}$. In fact, $\chi^{2} / N_{\mathrm{DF}}$ is unacceptably large as $\chi^{2} / N_{\mathrm{DF}}=10.1$ at $\beta=5.7, \chi^{2} / N_{\mathrm{DF}}=13.7$ at $\beta=5.8$ even for the best fit. (Of course, when $\sigma_{\Delta}$ is fixed to be the half value of the string tension in the $\mathrm{Q}-\overline{\mathrm{Q}}$ potential as in Refs. 26, 27, 28, 29], this fit with the $\Delta$-ansatz becomes further worse with a larger $\chi^{2} / N_{\mathrm{DF}}$.)

As an approximation, however, $V_{3 \mathrm{Q}}$ seems described by a simple sum of the effective two-body $\mathrm{Q}-\mathrm{Q}$ potential with a reduced string tension as

$$
\sigma_{\Delta} \simeq 0.53 \sigma_{\mathrm{Q} \overline{\mathrm{Q}}}
$$

This reduction factor can be naturally understood as a geometrical factor rather than the color factor, since the ratio between $L_{\min }$ and the perimeter length $L_{P}$ of the $3 \mathrm{Q}$ triangle satisfies

$$
\frac{1}{2} \leq \frac{L_{\min }}{L_{P}} \leq \frac{1}{\sqrt{3}}
$$

which leads to $L_{\min } \sigma=L_{P} \sigma_{\mathrm{QQ}}$ with $\sigma_{\mathrm{QQ}}=(0.5 \sim 0.58) \sigma$. The OGE relation is also found as $A_{\Delta} \simeq \frac{1}{2} A_{\mathrm{Q} \overline{\mathrm{Q}}}$.

For the fair comparison, we also examine the fit analysis by $\Delta$-ansatz with the lattice Coulomb potential, as was done for the Y-ansatz in Section 5-5. The 3Q data are fitted by the form of

$$
V_{3 \mathrm{Q}}=-A_{\Delta}^{\mathrm{LC}} \sum_{i<j} V^{\mathrm{LC}}\left(\overrightarrow{n_{i j}}\right)+\sigma_{\Delta}^{\mathrm{LC}} \sum_{i<j}\left|\mathbf{r}_{i}-\mathbf{r}_{j}\right|+C_{\Delta}^{\mathrm{LC}} .
$$

We add the results in Table 12. Again, a reduction in $\chi^{2} / N_{\mathrm{DF}}$ is observed. The values of the best-fit parameters are almost unchanged, and hence Eq.(41) and the OGE relation also hold. However, in comparison with the Y-ansatz with the lattice Coulomb potential, $\chi^{2} / N_{\mathrm{DF}}$ in this fit with the $\Delta$-ansatz is still larger, and therefore the $\Delta$-ansatz is difficult to be accepted.

\subsection{A more general ansatz - the generalized Y-ansatz}

From the theoretical reason of the short-distance perturbative QCD and the large-distance strongcoupling QCD, the Y-ansatz seems reasonable in the both limits. The overall lattice QCD data for the 3Q potential also support the $\mathrm{Y}$-ansatz rather than $\Delta$ ansatz. However, it is not trivial whether the Y-ansatz holds in the intermediate region as $0.2 \mathrm{fm}<r_{i j}<0.8 \mathrm{fm}$. In fact, as was conjectured by Conwall[30], there is a possibility of the $\Delta$-ansatz contamination in this region, where strong-coupling QCD is not applicable. In addition, a few recent lattice works seem to support the $\Delta$-ansatz for the 3Q potential in the intermediate region, although their studies were performed only for the equilateral triangle $3 \mathrm{Q}$ configuration. Of course, it is rather difficult to analyze the short distance behavior of the non-Coulomb part of the $3 \mathrm{Q}$ potential, because the Coulomb part is dominant there. Furthermore, the Coulomb potential form itself is no more trivial in the intermediate region as $0.2 \mathrm{fm}<r_{i j}<0.8 \mathrm{fm}$, where the perturbative QCD would not be valid.

In this section, we investigate the lattice QCD data for the $3 \mathrm{Q}$ potential using a more general ansatz which includes both the $\mathrm{Y}$ and the $\Delta$ ansätze in some limits. On the adoption of the general ansatz, we consider the possibility of the flux-tube core effect. For instance, in the dual superconductor 
picture 12, 13, 37, 38], the hadron flux tube has an intrinsic structure of the core region inside, In fact, if there exists the flux-tube core with the cylindrical radius $R_{\text {core }}$, the Y-type flux tube may be almost identical to the $\Delta$-type configuration at the short distance as $r_{i j} \sim R_{\text {core }}$, and thus the flux-tube length becomes obscured there.

So far, we have defined the minimal flux-tube length, which is proportional to the linear potential, as

$$
L_{\text {min }} \equiv P Q_{1}+P Q_{2}+P Q_{3}
$$

with the Fermat point $P$ in Fig. 14. In this section, considering a possible flux-tube core effect, we introduce the modified minimal flux-tube length $\bar{L}_{\text {min }}$ defined as

$$
\overline{L_{\min }} \equiv \frac{1}{2}\left(\overline{Q_{1} P_{2} Q_{3}}+\overline{Q_{2} P_{3} Q_{1}}+\overline{Q_{3} P_{1} Q_{2}}\right),
$$

with $\overline{A B C} \equiv \overline{A B}+\overline{B C}$, as shown in Fig. 14. Here, the points $P_{k}(k=1,2,3)$ are taken inside the circle centered at the Fermat point $P$ with the radius $R_{\text {core }}$, and $P_{1}$ is chosen so as to minimize

$$
\overline{Q_{3} P_{1} Q_{2}} \equiv \overline{P_{1} Q_{2}}+\overline{P_{1} Q_{3}}
$$

and so on. In this definition, when the circle crosses or includes the line $Q_{2} Q_{3}, P_{1}$ can be taken on the line $Q_{2} Q_{3}$ and then one finds

$$
\overline{Q_{3} P_{1} Q_{2}}=\overline{Q_{2} Q_{3}} .
$$

It is worth mentioning that there are two special cases corresponding to the $\mathrm{Y}$ and the $\Delta$ ansätze:

$$
\overline{L_{\min }}=L_{\min }
$$

in the case of $R_{\text {core }}=0$ or $R_{\text {core }} \ll r_{i j}$, and

$$
\overline{L_{\min }}=\frac{1}{2}\left(\overline{Q_{1} Q_{2}}+\overline{Q_{2} Q_{3}}+\overline{Q_{3} Q_{1}}\right)
$$

in the limit of $R_{\text {core }}=\infty$ or $R_{\text {core }} \gg r_{i j}$.

Using this modified minimal flux-tube length $\bar{L}_{\text {min }}$, we define the generalized Y-ansatz as

$$
V_{3 \mathrm{Q}}=\sigma_{\mathrm{GY}} \overline{L_{\mathrm{min}}}-A_{\mathrm{GY}} \sum_{i<j} \frac{1}{\left|\mathbf{r}_{i}-\mathbf{r}_{j}\right|}+C_{\mathrm{GY}} .
$$

This generalized Y-ansatz includes both the $\mathrm{Y}$ and the $\Delta$ ansätze in the special cases of $R_{\text {core }}=0$ and $R_{\text {core }}=\infty$, respectively. In fact, the generalized Y-ansatz reproduces the Y-ansatz at the large distance, which is theoretically supported by strong-coupling QCD, with including the $\Delta$-ansatz behavior at the short distance.

Then, we investigate the fit analysis for the lattice QCD data of the 3Q potential using the generalized Y-ansatz with varying $R_{\text {core }}$. We show in Table 13 the result of the fit analysis at $\beta=5.8$ and 6.0 . We observe the best fitting at $R_{\text {core }} \simeq 0.08 \mathrm{fm}$ both at $\beta=5.8$ and 6.0 . The values of the best-fit parameters $\left(\sigma_{\mathrm{GY}}, A_{\mathrm{GY}}, C_{\mathrm{GY}}\right)$ are almost the same as those in the Y-ansatz. This result also seems to support the Y-ansatz rather than the $\Delta$ ansatz at the hadronic scale as $r \gg 0.1 \mathrm{fm}$. (As an interesting speculation, $R_{\text {core }} \simeq 0.08 \mathrm{fm}$, which is almost the same both at $\beta=5.8$ and 6.0 , may physically correspond to the flux-tube core radius in the dual superconductor picture.)

\section{Summary and Concluding Remarks}

We have studied the static three-quark (3Q) potential in detail using SU(3) lattice QCD with $12^{3} \times 24$ at $\beta=5.7$ and $16^{3} \times 32$ at $\beta=5.8,6.0$ at the quenched level. In the first half of this paper, we have performed accurate measurement of the $3 \mathrm{Q}$ Wilson loop with the smearing technique, which reduces excited-state contaminations, and have presented the lattice QCD data of the 3Q ground-state potential $V_{3 \mathrm{Q}}$ for more than 300 different patterns of the $3 \mathrm{Q}$ systems. 
In the latter half, we have investigated the fit analysis on $V_{3 \mathrm{Q}}$, and have found that the lattice QCD data of the $3 \mathrm{Q}$ potential $V_{3 \mathrm{Q}}$ are well reproduced within a few $\%$ deviation by the sum of a constant, the two-body Coulomb term and the three-body linear confinement term $\sigma_{3 \mathrm{Q}} L_{\min }$, with $L_{\text {min }}$ the minimal value of the total length of color flux tubes linking the three quarks. We have investigated also the fit analysis with the lattice Coulomb potential instead of the Coulomb potential, and have found a better fit with keeping the similar result. From the comparison with the Q-Q potential, we have found a universality of the string tension as $\sigma_{3 \mathrm{Q}} \simeq \sigma_{\mathrm{QQ}}$ and the one-gluon-exchange result for the Coulomb coefficients as $A_{3 \mathrm{Q}} \simeq \frac{1}{2} A_{\mathrm{Q}}$.

We have also performed the various fit analyses. Through the fit with the Yukawa potential based on the Y-ansatz, we have observed no definite evidence that the short-distance potential becomes the Yukawa potential. The fit with the $\Delta$-ansatz is worse than that with the Y-ansatz on the confinement part in the $3 \mathrm{Q}$ potential $V_{3 \mathrm{Q}}$, although $V_{3 \mathrm{Q}}$ seems to be approximated by the $\Delta$-ansatz with $\sigma_{\Delta} \simeq 0.53 \sigma$. We have considered a more general ansatz including the $\mathrm{Y}$ and the $\Delta$ ansätze in some limits, and have found a possibility that the Y-type flux tube has a flux-tube core about $0.08 \mathrm{fm}$, which may appear as a small mixing of the $\Delta$-ansatz at the short distance, although such a small $\Delta$-type contamination is negligible in the intermediate and the infrared regions. To conclude, all of these detailed fit analyses for the lattice QCD data of the 3Q potential support the Y-ansatz.

\section{Acknowledgement}

We thank Dr. T. Umeda for his useful comments on the programming technique. H.S. is supported by Grant for Scientific Research (No.12640274) from Ministry of Education, Culture, Science and Technology, Japan. H. M. is supported by Japan Society for the Promotion of Science for Young Scientists. The lattice QCD Monte Carlo calculations have been performed on NEC-SX4 and NEC-SX5 at Osaka University and on HITACHI-SR8000 at KEK.

\section{References}

[1] M. Creutz, "Quarks, Gluons and Lattices" (Cambridge, 1983) 1.

[2] H. J. Rothe, "Lattice Gauge Theories" (World Scientific, 1992) 1.

[3] T.T. Takahashi, H. Matsufuru, Y. Nemoto and H. Suganuma, Phys. Rev. Lett. 86, 18 (2001).

[4] H. Matsufuru, Y. Nemoto, H. Suganuma, T. T. Takahashi and T. Umeda, Nucl. Phys. B (Proc. Suppl.) 94, 554 (2001).

[5] G.S. Bali, C. Schlichter and K. Schilling, Phys. Rev. D51, 5165 (1995).

[6] Y. Nambu, Phys. Rev. D10, 4262 (1974).

[7] J. Kogut and L. Susskind, Phys. Rev. D11, 395 (1975).

[8] A. Casher, H. Neuberger and S. Nussinov, Phys. Rev. D20, 179 (1979).

[9] J. Carlson, J.B. Kogut and V.R. Pandharipande, Phys. Rev. D27, 233 (1983); D28,2807 (1983).

[10] S. Capstick and N. Isgur, Phys. Rev. D34 2809, (1986).

[11] N. Isgur and J. Paton, Phys. Lett. B124, 247 (1983); Phys. Rev. D31, 2910 (1985).

[12] H. Suganuma, S. Sasaki and H. Toki, Nucl. Phys. B435, 207 (1995).

[13] H. Suganuma, S. Sasaki, H. Toki and H. Ichie, Prog. Theor. Phys. Suppl. 120, 57(1995).

[14] W. Greiner and A. Schäfer, "Quantum Chromodynamics" (Springer, 1994) 1.

[15] K. Sailer, Th. Schonfeld, Zs. Schram, A. Schafer and W. Greiner, J. Phys. G: Nucl. Part. Phys. 17, 1005 (1991), and references therein. 
[16] W. Lucha, F.F. Schöberl and D. Gromes, Phys. Rep. 200, 127 (1991).

[17] A. Di Giacomo, M. Maggiore and S. Olejnik, Phys. Lett. B236, 199 (1990); Nucl. Phys. B347, 441 (1990).

[18] Y.-C. Peng, R.W. Haymaker, Phys. Rev. D47, 5104 (1993).

[19] R.W. Haymaker, V. Singh, Y.-C. Peng and J. Wosiek, Phys. Rev. D53, 389 (1996).

[20] N. Brambilla, G.M. Prosperi and A. Vairo, Phys. Lett. B362, 113 (1995).

[21] M. Fable de la Ripelle and Yu. A. Simonov, Ann. Phys. 212, 235 (1991).

[22] G.S. Bali and K. Schilling, Phys. Rev. D46, 2636 (1992).

[23] K. Schilling, Nucl. Phys. B (Proc.Suppl.) 83-84, 140 (2000) and references therein.

[24] R. Sommer and J. Wosiek, Phys. Lett. 149B,497 (1984); Nucl. Phys. B267, 531 (1986).

[25] J. Kamesberger, G. Eder, M.E. Faber, H. Leeb and H. Markum, Proc. of "Few-Body Problems in Particle, Nuclear, Atomic and Molecular Physics" (1987) 529.

[26] H.B. Thacker, E. Eichten and J.C. Sexton, Nucl. Phys. B (Proc. Suppl.) 4, 234 (1988).

[27] G. S. Bali, Phys. Rept. 343, 1 (2001).

[28] C. Alexandrou, Ph. de Forcrand and A. Tsapalis, Nucl. Phys. B (Proc. Suppl.) 106, 403 (2002).

[29] C. Alexandrou, Ph. de Forcrand and A. Tsapalis, Phys. Rev. D65, 054503 (2002).

[30] J.M. Cornwall, Phys. Rev. D54, 6527 (1996).

[31] T. T. Takahashi, H. Matsufuru, Y. Nemoto and H. Suganuma, Proc. of the Int. Symp. on "Dynamics of Gauge Fields", Tokyo, Dec. 13-15, 1999, edited by A. Chodos, N. Kitazawa, H. Minakata and C.M. Sommerfield, (Universal Academy Press, 2000) 179.

[32] H. Suganuma, H. Matsufuru, Y. Nemoto and T.T. Takahashi, Nucl. Phys. A680, 159 (2000).

[33] T. T. Takahashi, H. Suganuma, H. Matsufuru and Y. Nemoto, AIP Conference Proceedings CP594, 341 (2001): Proc. of Int. Symp. on "Hadrons and Nuclei", Seoul, Korea, Feb. 20-22, 2001, edited by I.-T. Cheon and T.K. Choi.

[34] APE Collaboration, M. Albanese et al., Phys. Lett. B192, 163 (1987).

[35] N. Ishii, H. Suganuma and H. Matsufuru, preprint hep-lat/0109011; Nucl. Phys. B (Proc. Suppl.) 106, $516(2002)$.

[36] I. Montvay and G. Münster, "Quantum Fields on a Lattice" (Cambridge, 1994) 389.

[37] "Color Confinement and Hadrons", edited by H. Toki, Y. Mizuno, H. Suganuma, T. Suzuki and O. Miyamura, (World Scientific, 1995) 1.

[38] "Quantum Chromodynamics and Color Confinement", edited by H. Suganuma, M. Fukushima and H. Toki, (World Scientific, 2001) 1.

[39] H. Suganuma, K. Amemiya, H. Ichie and A. Tanaka, Nucl. Phys. A670, 40 (2000).

[40] H. Suganuma, H. Ichie, A. Tanaka and K. Amemiya, Prog. Theor. Phys. Suppl. 131, 559 (1998).

[41] G. Feinberg and J. Sucher, Phys. Rev. D20, 1717 (1979).

[42] M. Oka and K. Yazaki, Prog. Theor. Phys. 66, 556 (1981); ibid. 572 (1981). 
Figure 1: The flux-tube configuration of the 3Q system with the minimal value of the total flux-tube length. There appears a physical junction linking the three flux tubes at the Fermat point P.

Figure 2: The $3 \mathrm{Q}$ Wilson loop $W_{3 \mathrm{Q}}$. The $3 \mathrm{Q}$ state is generated at $t=0$ and is annihilated at $t=T$. The three quarks are spatially fixed in $\mathbf{R}^{3}$ for $0<t<T$.

Figure 3: The schematic explanation of the smearing for the link-variables. 
Figure 4: The schematic explanation of the physical meaning of the smeared line. The $n$-th smeared line depicted as (a) physically corresponds to the spatially-distributed flux tube as (b) in terms of the original field variable. The single smearing procedure for the line is illustrated with (c) on the lattice. The flux perpendicular to the line is expected to be canceled. The spatial distribution of the $n$-th smeared line is expressed by the Gaussian profile $\phi(r)$ with $r=\left(x^{2}+y^{2}\right)^{1 / 2}$ as shown in (d).

Figure 5: The ground-state overlap of the 3Q system, $C_{0} \equiv\left\langle W_{3 \mathrm{Q}}(T)\right\rangle^{T+1} /\left\langle W_{3 \mathrm{Q}}(T+1)\right\rangle^{T}$, with the smeared link-variable (upper data) and with unsmeared link-variable (lower data) at $\beta=5.7$. To distinguish the $3 \mathrm{Q}$ system, we have taken the horizontal axis as $L_{\min }$, which denotes the minimal value of the total length of the flux tubes linking the three quarks. For each $3 \mathrm{Q}$ configuration, $C_{0}$ is largely enhanced as $0.8<C_{0}<1$ by the smearing. 
Figure 6: The $\mathrm{Q} \overline{\mathrm{Q}}$ static potential $V_{\mathrm{Q} \overline{\mathrm{Q}}}(r)$ as the function of the inter-quark distance $r$ in the lattice unit in $\mathrm{SU}(3)$ lattice $\mathrm{QCD}$ with $\beta=6.0$ at the quenched level.

Figure 7: The lattice QCD data for the $3 \mathrm{Q}$ potential $V_{3 \mathrm{Q}}^{\text {latt }}$ at $\beta=5.7$ as the function of $L_{\min }$, the minimum value of the total length of the flux tubes, in the lattice unit. 
Figure 8: The lattice QCD data for the $3 \mathrm{Q}$ potential $V_{3 \mathrm{Q}}^{\text {latt }}$ at $\beta=5.8$ as the function of $L_{\mathrm{min}}$, the minimum value of the total length of the flux tubes, in the lattice unit.

Figure 9: The lattice QCD data for the $3 \mathrm{Q}$ potential $V_{3 \mathrm{Q}}^{\text {latt }}$ at $\beta=6.0$ as the function of $L_{\min }$, the minimum value of the total length of the flux tubes, in the lattice unit. 
Figure 10: The semi-quantitative test on the confinement part in the $3 \mathrm{Q}$ potential $V_{3 \mathrm{Q}}$ at $\beta=5.8$. The Coulomb-subtracted potential $V_{3 \mathrm{Q}}^{\text {latt }}-V_{3 \mathrm{Q}}^{\text {Coul }}$ is plotted as the function of $L_{\min }$, the minimal value of the total flux-tube length. Here, the Coulomb part $V_{3 \mathrm{Q}}^{\text {Coul }}$ is evaluated from the $\mathrm{Q}-\overline{\mathrm{Q}}$ potential.

Figure 11: The semi-quantitative test on the confinement part in the $3 \mathrm{Q}$ potential $V_{3 \mathrm{Q}}$ at $\beta=6.0$. The Coulomb-subtracted potential $V_{3 \mathrm{Q}}^{\text {latt }}-V_{3 \mathrm{Q}}^{\text {Coul }}$ is plotted as the function of $L_{\min }$, the minimal value of the total flux-tube length. Here, the Coulomb part $V_{3 \mathrm{Q}}^{\text {Coul }}$ is evaluated from the $\mathrm{Q}-\overline{\mathrm{Q}}$ potential. 
Figure 12: The comparison between the lattice QCD data $V_{3 \mathrm{Q}}^{\text {latt }}$ at $\beta=5.7$ and the fitted curve of $V_{3 \mathrm{Q}}^{\mathrm{fit}}$ as the function of $i$ for each $(j, k)$ fixed, when the three quarks are located at $(i, 0,0),(0, j, 0),(0,0, k)$ in the lattice unit. The lattice data $V_{3 \mathrm{Q}}^{\text {latt }}$ are expressed as the points, and $V_{3 \mathrm{Q}}^{\text {fit }}$ is expressed as the solid curve for each $(j, k)$.

Figure 13: The comparison of the lattice Coulomb (LC) potential $V^{\mathrm{LC}}(\vec{n})$ with $1 / r$ in the lattice unit. We plot the on-axis data $V^{\mathrm{LC}}(k, 0,0)(0 \leq k \leq 7)$ by the closed circle, the off-axis data $V^{\mathrm{LC}}(k, k, 0)$ $(1 \leq k \leq 4)$ by the open circle, and $1 / r$ by the solid curve. 
Figure 14: The visual illustration of the generalized Y-ansatz. The three quarks are spatially fixed at $Q_{1}, Q_{2}$ and $Q_{3}$. The circle around the Fermat point $\mathrm{P}$ corresponds to the flux-tube core around the physical junction. The point $\mathrm{P}_{1}$ is taken inside the circle so as to minimize $\mathrm{P}_{1} \mathrm{Q}_{2}+\mathrm{P}_{1} \mathrm{Q}_{3}$, and $\mathrm{P}_{2}$ and $\mathrm{P}_{3}$ are similarly defined.

\begin{tabular}{cccccccccc}
\hline \hline$\beta$ & $a[\mathrm{fm}]$ & lattice size & $N_{3 \mathrm{Q}}$ & $N_{\text {conf }}$ & $N_{\text {therm }}$ & $N_{\text {sep }}$ & $\alpha$ & $N_{\text {smr }}$ & super-computer \\
\hline 5.7 & 0.19 & $12^{3} \times 24$ & 16 & 210 & 5,000 & 500 & 2.3 & 12 & NEC SX-4 \\
5.8 & 0.14 & $16^{3} \times 32$ & 139 & 200 & 10,000 & 500 & 2.3 & 22 & HITACHI SR8000 \\
6.0 & 0.10 & $16^{3} \times 32$ & 155 & 150 & 10,000 & 500 & 2.3 & 42 & NEC SX-5 \\
\hline \hline
\end{tabular}

Table 1: The simulation condition and the related information. For each $\beta$, the corresponding lattice spacing $a$, the lattice size, the number $N_{3 \mathrm{Q}}$ of the different patterns of the $3 \mathrm{Q}$ system analyzed, the number $N_{\text {conf }}$ of the gauge configuration used for the measurement, the number $N_{\text {therm }}$ of sweeps for the thermalization, the number $N_{\text {sep }}$ of sweeps for the separation, the smearing parameter $\alpha$, the iteration number $N_{\mathrm{smr}}$ of the suitable smearing for the $3 \mathrm{Q}$ potential, and the used super-computer are listed. 


\begin{tabular}{lcccl}
\hline \hline & $\sigma$ & $A$ & $C$ & $\chi^{2} / N_{\mathrm{DF}}$ \\
\hline $3 \mathrm{Q}_{\mathrm{Y}}$ & $0.1524(28)$ & $0.1331(66)$ & $0.9182(213)$ & 3.76 \\
$3 \mathrm{Q}_{\mathrm{Y}}$ (Latt. Coul.) & $0.1556(24)$ & $0.1185(53)$ & $0.8876(179)$ & 1.81 \\
\hline $\mathrm{Q} \overline{\mathrm{Q}}$ (on-axis) & $0.1629(47)$ & $0.2793(116)$ & $0.6203(161)$ & 0.59 \\
$\mathrm{Q} \overline{\mathrm{Q}}$ (on-axis, Latt. Coul.) & $0.1603(48)$ & $0.2627(109)$ & $0.6271(165)$ & 0.51 \\
$\mathrm{Q} \overline{\mathrm{Q}}$ (off-axis, Latt. Coul.) & $0.1611(18)$ & $0.2780(44)$ & $0.6430(63)$ & 3.57 \\
\hline \hline
\end{tabular}
$\beta=5.7$ (16 quark configurations)

\begin{tabular}{lcccl}
\hline \hline & $\sigma$ & $A$ & $C$ & $\chi^{2} / N_{\mathrm{DF}}$ \\
\hline $3 \mathrm{Q}_{\mathrm{Y}}$ & $0.1027(6)$ & $0.1230(20)$ & $0.9085(55)$ & 5.03 \\
$3 \mathrm{Q}_{\mathrm{Y}}$ (Latt. Coul.) & $0.1031(6)$ & $0.1141(18)$ & $0.8999(54)$ & 4.29 \\
\hline $\mathrm{Q} \overline{\mathrm{Q}}$ (on-axis) & $0.1079(28)$ & $0.2607(174)$ & $0.6115(197)$ & 0.92 \\
$\mathrm{Q} \overline{\mathrm{Q}}$ (on-axis, Latt. Coul.) & $0.1080(28)$ & $0.2377(159)$ & $0.6074(194)$ & 0.76 \\
$\mathrm{Q} \overline{\mathrm{Q}}$ (off-axis, Latt. Coul.) & $0.1018(11)$ & $0.2795(51)$ & $0.6596(53)$ & 1.28 \\
\hline \hline
\end{tabular}
$\beta=5.8$ (139 quark configurations)

\begin{tabular}{lcccl}
\hline \hline & $\sigma$ & $A$ & $C$ & $\chi^{2} / N_{\mathrm{DF}}$ \\
\hline $3 \mathrm{Q}_{\mathrm{Y}}$ & $0.0460(4)$ & $0.1366(11)$ & $0.9599(35)$ & 2.81 \\
$3 \mathrm{Q}_{\mathrm{Y}}$ (Latt. Coul.) & $0.0467(4)$ & $0.1256(10)$ & $0.9467(34)$ & 2.22 \\
\hline $\mathrm{Q} \overline{\mathrm{Q}}$ (on-axis) & $0.0506(7)$ & $0.2768(24)$ & $0.6374(30)$ & 3.56 \\
$\mathrm{Q} \overline{\mathrm{Q}}$ (on-axis, Latt. Coul.) & $0.0500(7)$ & $0.2557(22)$ & $0.6373(30)$ & 1.22 \\
$\mathrm{Q} \overline{\mathrm{Q}}$ (off-axis, Latt. Coul.) & $0.0497(5)$ & $0.2572(15)$ & $0.6389(20)$ & 1.59 \\
\hline \hline
\end{tabular}

$\beta=6.0$ (155 quark configurations)

Table 2: The main result on the fit analysis of the lattice QCD data with the Y-ansatz at each $\beta$. We list the best-fit parameter set $(\sigma, A, C)$ in the function form as $V_{3 \mathrm{Q}}=-A_{3 \mathrm{Q}} \sum_{i<j} \frac{1}{\left|\mathbf{r}_{i}-\mathbf{r}_{j}\right|}+\sigma_{3 \mathrm{Q}} L_{\min }+C_{3 \mathrm{Q}}$, where $L_{\text {min }}$ denotes the minimal value of the Y-type flux-tube length. The label of (Latt. Coul.) means the fit with the lattice Coulomb potential instead of the continuum Coulomb potential. The similar fit on the Q-Q potential is also listed: "on-axis" and "off-axis" mean the fit analysis only for on-axis data and for both on-axis and off-axis data, respectively. The universality of the string tension and the OGE result on the Coulomb coefficient are found as $\sigma_{3 \mathrm{Q}} \simeq \sigma_{\mathrm{QQ}}$ and $A_{3 \mathrm{Q}} \simeq A_{\mathrm{Q} \overline{\mathrm{Q}}}$, respectively. The listed values are measured in the lattice unit. 


\begin{tabular}{ccccccr}
\hline \hline$(i, j, k)$ & $V_{3 \mathrm{Q}}^{\text {latt }}$ & $\bar{C}$ & fit range of $T$ & $\chi^{2} / N_{\mathrm{DF}}$ & $V_{3 \mathrm{Q}}^{\text {fit }}$ & $V_{3 \mathrm{Q}}^{\text {latt }}-V_{3 \mathrm{Q}}^{\text {fit }}$ \\
\hline$(0,1,1)$ & $0.8457(38)$ & $0.9338(173)$ & $5-10$ & 0.062 & 0.8524 & -0.0067 \\
$(0,1,2)$ & $1.0973(43)$ & $0.9295(161)$ & $4-8$ & 0.163 & 1.1025 & -0.0052 \\
$(0,1,3)$ & $1.2929(41)$ & $0.8987(110)$ & $3-7$ & 0.255 & 1.2929 & 0.0000 \\
$(0,2,2)$ & $1.3158(44)$ & $0.9151(120)$ & $3-6$ & 0.053 & 1.3270 & -0.0112 \\
$(0,2,3)$ & $1.5040(63)$ & $0.9041(170)$ & $3-6$ & 0.123 & 1.5076 & -0.0036 \\
$(0,3,3)$ & $1.6756(43)$ & $0.8718(73)$ & $2-5$ & 0.572 & 1.6815 & -0.0059 \\
$(1,1,1)$ & $1.0238(40)$ & $0.9345(149)$ & $4-8$ & 0.369 & 1.0092 & 0.0146 \\
$(1,1,2)$ & $1.2185(62)$ & $0.9067(228)$ & $4-8$ & 0.352 & 1.2151 & 0.0034 \\
$(1,1,3)$ & $1.4161(49)$ & $0.9297(135)$ & $3-7$ & 0.842 & 1.3964 & 0.0197 \\
$(1,2,2)$ & $1.3866(48)$ & $0.9012(127)$ & $3-7$ & 0.215 & 1.3895 & -0.0029 \\
$(1,2,3)$ & $1.5594(63)$ & $0.8880(165)$ & $3-6$ & 0.068 & 1.5588 & 0.0006 \\
$(1,3,3)$ & $1.7145(43)$ & $0.8553(76)$ & $2-6$ & 0.412 & 1.7202 & -0.0057 \\
$(2,2,2)$ & $1.5234(37)$ & $0.8925(65)$ & $2-5$ & 0.689 & 1.5238 & -0.0004 \\
$(2,2,3)$ & $1.6750(118)$ & $0.8627(298)$ & $3-6$ & 0.115 & 1.6763 & -0.0013 \\
$(2,3,3)$ & $1.8239(56)$ & $0.8443(90)$ & $2-5$ & 0.132 & 1.8175 & 0.0064 \\
$(3,3,3)$ & $1.9607(93)$ & $0.8197(154)$ & $2-5$ & 0.000 & 1.9442 & 0.0165 \\
\hline \hline
\end{tabular}

Table 3: Lattice QCD results for the 3Q potential $V_{3 \mathrm{Q}}^{\text {latt }}$ in the lattice unit for 16 patterns of the 3Q system at $\beta=5.7$. $(i, j, k)$ denotes the $3 \mathrm{Q}$ system where the three quarks are put on $(i, 0,0),(0, j, 0)$ and $(0,0, k)$ in $\mathbf{R}^{3}$ in the lattice unit. For each $3 \mathrm{Q}$ configuration, $V_{3 \mathrm{Q}}^{\text {latt }}$ is measured from the singleexponential fit as $\left\langle W_{3 \mathrm{Q}}\right\rangle=\bar{C} e^{-V_{3 \mathrm{Q}} T}$ in the range of $T$ listed at the fourth column. The statistical errors listed are estimated with the jackknife method, and $\chi^{2} / N_{\mathrm{DF}}$ is listed at the fifth column. The best-fit function $V_{3 \mathrm{Q}}^{\mathrm{fit}}$ in the $\mathrm{Y}$-ansatz is added. 
Table 4: A part of lattice QCD results for the $3 \mathrm{Q}$ potential $V_{3 \mathrm{Q}}^{\text {latt }}$ at $\beta=5.8 .(i, j, k)$ denotes the $3 \mathrm{Q}$ system where the three quarks are put on $(i, 0,0)$, $(0, j, 0)$ and $(0,0, k)$ in $\mathbf{R}^{3}$ in the lattice unit. For each $3 \mathrm{Q}$ configuration, $V_{3 \mathrm{Q}}^{\text {latt }}$ is measured from the single-exponential fit as $\left\langle W_{3 \mathrm{Q}}\right\rangle=\bar{C} e^{-V_{3 \mathrm{Q}} T}$. The prefactor $\bar{C}$ physically means the magnitude of the ground-state component. The difference from the best-fit function $V_{3 \mathrm{Q}}^{\mathrm{fit}}$ in the $\mathrm{Y}$-ansatz is added. The listed values are measured in the lattice unit.

\begin{tabular}{cccc}
\hline \hline$(i, j, k)$ & $V_{3 \mathrm{Q}}^{\text {latt }}$ & $\bar{C}$ & $V_{3 \mathrm{Q}}^{\text {latt }}-V_{3 \mathrm{Q}}^{\text {fit }}$ \\
\hline$(0,1,1)$ & $0.7697(12)$ & $0.9554(58)$ & -0.0041 \\
$(0,1,2)$ & $0.9639(28)$ & $0.9269(128)$ & -0.0039 \\
$(0,1,3)$ & $1.1112(60)$ & $0.9274(274)$ & 0.0053 \\
$(0,1,4)$ & $1.2337(119)$ & $0.9106(538)$ & 0.0064 \\
$(0,1,5)$ & $1.3219(235)$ & $0.7957(927)$ & -0.0195 \\
$(0,1,6)$ & $1.4518(132)$ & $0.8583(442)$ & 0.0000 \\
$(0,1,7)$ & $1.5719(72)$ & $0.8798(182)$ & 0.0119 \\
$(0,1,8)$ & $1.6621(105)$ & $0.8189(261)$ & -0.0048 \\
$(0,2,2)$ & $1.1370(16)$ & $0.9342(46)$ & -0.0018 \\
$(0,2,3)$ & $1.2659(21)$ & $0.9145(52)$ & -0.0027 \\
$(0,2,4)$ & $1.3585(239)$ & $0.7975(946)$ & -0.0279 \\
$(0,2,5)$ & $1.4834(128)$ & $0.8448(431)$ & -0.0152 \\
$(0,2,6)$ & $1.6082(28)$ & $0.8810(50)$ & 0.0004 \\
$(0,2,8)$ & $1.8251(132)$ & $0.8548(332)$ & 0.0036 \\
$(0,3,3)$ & $1.3925(91)$ & $0.9168(330)$ & -0.0003 \\
$(0,3,4)$ & $1.5005(41)$ & $0.8862(109)$ & -0.0066 \\
$(0,3,5)$ & $1.6130(25)$ & $0.8810(41)$ & -0.0042 \\
$(0,3,6)$ & $1.7171(32)$ & $0.8581(54)$ & -0.0080 \\
$(0,4,4)$ & $1.6077(72)$ & $0.8655(185)$ & -0.0112 \\
$(0,4,5)$ & $1.7163(32)$ & $0.8581(52)$ & -0.0109 \\
$(0,4,6)$ & $1.8262(40)$ & $0.8482(67)$ & -0.0075 \\
$(0,4,7)$ & $1.9321(51)$ & $0.8317(82)$ & -0.0070 \\
$(0,4,8)$ & $2.0413(65)$ & $0.8202(101)$ & -0.0025 \\
$(0,5,5)$ & $1.8193(44)$ & $0.8372(72)$ & -0.0147 \\
$(0,5,6)$ & $1.9282(47)$ & $0.8265(76)$ & -0.0111 \\
$(0,5,8)$ & $2.1460(73)$ & $0.8047(113)$ & -0.0017 \\
$(0,6,6)$ & $2.0322(62)$ & $0.8083(102)$ & -0.0113 \\
$(0,6,7)$ & $2.1401(71)$ & $0.7964(112)$ & -0.0070 \\
$(0,6,8)$ & $2.2384(85)$ & $0.7698(123)$ & -0.0118 \\
$(0,7,7)$ & $2.2461(101)$ & $0.7813(153)$ & -0.0037 \\
$(0,7,8)$ & $2.3390(114)$ & $0.7462(166)$ & -0.0134 \\
$(0,8,8)$ & $2.4191(177)$ & $0.6949(244)$ & -0.0351 \\
$(1,1,1)$ & $0.9140(32)$ & $0.9424(147)$ & 0.0149 \\
$(1,1,2)$ & $1.0647(42)$ & $0.9290(194)$ & 0.0096 \\
$(1,1,3)$ & $1.1914(86)$ & $0.8917(384)$ & 0.0053 \\
$(1,1,4)$ & $1.2879(172)$ & $0.7887(674)$ & -0.0169 \\
$(1,1,5)$ & $1.4201(39)$ & $0.8662(104)$ & 0.0024 \\
$(1,1,6)$ & $1.5335(54)$ & $0.8600(136)$ & 0.0061 \\
$(1,1,7)$ & $1.6497(36)$ & $0.8686(59)$ & 0.0146 \\
$(1,1,8)$ & $1.7557(46)$ & $0.8509(76)$ & 0.0140 \\
$(1,2,2)$ & $1.1865(33)$ & $0.9186(120)$ & 0.0020 \\
\hline
\end{tabular}

Table 5: A part of lattice QCD results for the $3 \mathrm{Q}$ potential $V_{3 \mathrm{Q}}^{\text {latt }}$ at $\beta=5.8$. The notations are the same in Table 1 .

\begin{tabular}{|c|c|c|c|}
\hline$\overline{(i, j, k)}$ & $\overline{\overline{V_{3 \mathrm{Q}}^{\text {latt }}}}$ & $\overline{\bar{C}}$ & $V_{3 \mathrm{Q}}^{\text {latt }}-V_{3 \mathrm{Q}}^{\text {fit }}$ \\
\hline$(1,2,6)$ & $1.6356(57)$ & $0.8587(145)$ & -0.0031 \\
\hline$(1,2,7)$ & $1.7490(36)$ & 0.8591( 59) & 0.0035 \\
\hline$(1,2,8)$ & $1.8545(44)$ & $0.8407(70)$ & 0.0032 \\
\hline$(1,3,3)$ & $1.4175(33)$ & $0.9020(86)$ & -0.0024 \\
\hline$(1,3,4)$ & $1.5301(109)$ & $0.9083(391)$ & -0.0005 \\
\hline$(1,3,5)$ & $1.6272(50)$ & $0.8538(125)$ & -0.0116 \\
\hline$(1,3,6)$ & $1.7301(72)$ & $0.8285(178)$ & -0.0154 \\
\hline$(1,3,7)$ & $1.8300(105)$ & $0.7978(249)$ & -0.0213 \\
\hline$(1,3,8)$ & $1.9505(50)$ & $0.8196(78)$ & -0.0057 \\
\hline$(1,4,4)$ & $1.6284(60)$ & $0.8652(154)$ & -0.0099 \\
\hline$(1,4,5)$ & $1.7195(73)$ & $0.8133(173)$ & -0.0251 \\
\hline$(1,4,6)$ & $1.8213(90)$ & $0.7883(212)$ & -0.0286 \\
\hline$(1,4,7)$ & $1.9469(47)$ & $0.8216(72)$ & -0.0075 \\
\hline$(1,4,8)$ & $2.0543(59)$ & $0.8078(91)$ & -0.0043 \\
\hline$(1,5,5)$ & $1.8114(119)$ & $0.7692(271)$ & -0.0378 \\
\hline$(1,5,7)$ & $2.0496(55)$ & $0.8048(85)$ & -0.0071 \\
\hline$(1,5,8)$ & $2.1538(67)$ & $0.7863(100)$ & -0.0063 \\
\hline$(1,6,7)$ & $2.1642(71)$ & $0.8056(112)$ & 0.0055 \\
\hline$(1,6,8)$ & $2.2632(74)$ & $0.7794(113)$ & 0.0020 \\
\hline$(1,7,7)$ & $2.2808(92)$ & $0.8080(147)$ & 0.0203 \\
\hline$(1,7,8)$ & $2.3682(96)$ & $0.7642(141)$ & 0.0059 \\
\hline$(1,8,8)$ & $2.4512(156)$ & $0.7146(217)$ & -0.0123 \\
\hline$(2,2,2)$ & $1.2771(73)$ & $0.9000(258)$ & -0.0041 \\
\hline$(2,2,3)$ & $1.3783(80)$ & $0.8755(277)$ & -0.0107 \\
\hline$(2,2,4)$ & $1.4899(42)$ & $0.8768(107)$ & -0.0074 \\
\hline$(2,2,5)$ & $1.5933(54)$ & $0.8513(133)$ & -0.0113 \\
\hline$(2,2,8)$ & $1.9188(49)$ & $0.8210(79)$ & -0.0024 \\
\hline$(2,3,3)$ & $1.4739(120)$ & $0.8636(410)$ & -0.0142 \\
\hline$(2,3,4)$ & $1.5831(22)$ & $0.8718(36)$ & -0.0081 \\
\hline$(2,3,5)$ & $1.6820(57)$ & $0.8380(140)$ & -0.0133 \\
\hline$(2,3,6)$ & $1.7915(34)$ & $0.8361(58)$ & -0.0080 \\
\hline$(2,3,7)$ & $1.8982(43)$ & $0.8220(68)$ & -0.0053 \\
\hline$(2,3,8)$ & $2.0002(53)$ & $0.7989(85)$ & -0.0071 \\
\hline$(2,4,4)$ & $1.6805(77)$ & $0.8506(189)$ & -0.0102 \\
\hline$(2,4,5)$ & $1.7675(71)$ & $0.7952(166)$ & -0.0246 \\
\hline$(2,4,7)$ & $1.9863(48)$ & $0.7933(73)$ & -0.0107 \\
\hline$(2,4,8)$ & $2.0982(60)$ & $0.7875(94)$ & -0.0015 \\
\hline$(2,5,8)$ & $2.2004(72)$ & $0.7756(106)$ & 0.0051 \\
\hline$(2,6,6)$ & $2.0932(65)$ & $0.7907(94)$ & 0.0016 \\
\hline$(2,6,7)$ & $2.1928(71)$ & $0.7665(105)$ & 0.0010 \\
\hline$(2,6,8)$ & $2.2889(84)$ & $0.7378(120)$ & -0.0038 \\
\hline$(2,7,8)$ & $2.4014(106)$ & $0.7347(147)$ & 0.0103 \\
\hline$(3,3,3)$ & $1.5566(72)$ & $0.8434(180)$ & -0.0197 \\
\hline$(3,3,4)$ & $1.6474(66)$ & $0.8125(160)$ & -0.0253 \\
\hline$(3,3,5)$ & $1.7641(37)$ & $0.8353(60)$ & -0.0084 \\
\hline$(3,3,6)$ & $1.8685(44)$ & $0.8196(72)$ & -0.0053 \\
\hline$(3,3,7)$ & $1.9696(52)$ & $0.7965(82)$ & -0.0062 \\
\hline$(3,3,8)$ & $2.0753(65)$ & $0.7812(98)$ & -0.0029 \\
\hline$(3,4,5)$ & $1.8357(106)$ & $0.7690(240)$ & -0.0249 \\
\hline
\end{tabular}


Table 6: A part of lattice QCD results for the 3Q potential $V_{3 Q}^{\text {latt }}$ at $\beta=5.8$. The notations are the same in Table 1 .

\begin{tabular}{cccc}
\hline \hline$(i, j, k)$ & $V_{3 \mathrm{Q}}^{\text {latt }}$ & $\bar{C}$ & $V_{3 \mathrm{Q}}^{\text {latt }}-V_{3 \mathrm{Q}}^{\text {fit }}$ \\
\hline$(3,4,6)$ & $1.9440(157)$ & $0.7686(358)$ & -0.0153 \\
$(3,4,7)$ & $2.0569(60)$ & $0.7756(96)$ & -0.0025 \\
$(3,4,8)$ & $2.1666(68)$ & $0.7679(100)$ & 0.0063 \\
$(3,5,5)$ & $1.9321(178)$ & $0.7527(394)$ & -0.0220 \\
$(3,5,6)$ & $2.0504(56)$ & $0.7756(83)$ & -0.0004 \\
$(3,5,8)$ & $2.2627(77)$ & $0.7491(110)$ & 0.0138 \\
$(3,6,6)$ & $2.1580(69)$ & $0.7683(101)$ & 0.0123 \\
$(3,7,7)$ & $2.3578(110)$ & $0.7261(155)$ & 0.0190 \\
$(3,7,8)$ & $2.4496(125)$ & $0.6934(169)$ & 0.0133 \\
$(3,8,8)$ & $2.5416(178)$ & $0.6613(229)$ & 0.0088 \\
$(4,4,4)$ & $1.8377(49)$ & $0.8044(74)$ & -0.0119 \\
$(4,4,5)$ & $1.9371(55)$ & $0.7900(82)$ & -0.0044 \\
$(4,4,6)$ & $2.0367(61)$ & $0.7703(91)$ & -0.0004 \\
$(4,5,5)$ & $2.0278(68)$ & $0.7638(96)$ & -0.0022 \\
$(4,5,6)$ & $2.1301(69)$ & $0.7503(99)$ & 0.0073 \\
$(4,6,6)$ & $2.2310(87)$ & $0.7347(123)$ & 0.0174 \\
$(4,6,8)$ & $2.4356(117)$ & $0.7003(162)$ & 0.0322 \\
$(4,7,7)$ & $2.4304(130)$ & $0.6947(177)$ & 0.0306 \\
$(4,7,8)$ & $2.5347(150)$ & $0.6806(201)$ & 0.0402 \\
$(4,8,8)$ & $2.6412(245)$ & $0.6696(328)$ & 0.0531 \\
$(5,5,5)$ & $2.1192(87)$ & $0.7417(123)$ & 0.0050 \\
$(5,5,6)$ & $2.2247(90)$ & $0.7334(125)$ & 0.0208 \\
$(5,5,7)$ & $2.3298(104)$ & $0.7208(147)$ & 0.0328 \\
$(5,5,8)$ & $2.4280(131)$ & $0.6980(182)$ & 0.0357 \\
$(5,6,6)$ & $2.3159(114)$ & $0.7065(155)$ & 0.0252 \\
$(5,6,7)$ & $2.4124(125)$ & $0.6818(170)$ & 0.0307 \\
$(5,6,8)$ & $2.5255(151)$ & $0.6802(200)$ & 0.0503 \\
$(5,8,8)$ & $2.7193(345)$ & $0.6369(438)$ & 0.0660 \\
$(6,6,6)$ & $2.4166(223)$ & $0.6943(306)$ & 0.0421 \\
$(6,6,7)$ & $2.5096(190)$ & $0.6648(252)$ & 0.0468 \\
$(6,6,8)$ & $2.6408(230)$ & $0.6868(315)$ & 0.0866 \\
$(6,7,7)$ & $2.5846(264)$ & $0.6148(323)$ & 0.0358 \\
$(6,7,8)$ & $2.7365(260)$ & $0.6615(343)$ & 0.0981 \\
$(6,8,8)$ & $2.8742(456)$ & $0.6939(633)$ & 0.1479 \\
\hline \hline
\end{tabular}

Table 7: A part of lattice QCD results for the 3Q potential $V_{3 \mathrm{Q}}^{\text {latt }}$ at $\beta=5.8$. $(l, m, n)$ denotes the $3 \mathrm{Q}$ system where the three quarks are put on $(l, 0,0)$, $(-m, 0,0)$ and $(0, n, 0)$ in $\mathbf{R}^{3}$ in the lattice unit. The other notations are the same in Table 4.

\begin{tabular}{cccc}
\hline \hline$(l, m, n)$ & $V_{3 \mathrm{Q}}^{\text {latt }}$ & $\bar{C}$ & $V_{3 \mathrm{Q}}^{\text {latt }}-V_{3 \mathrm{Q}}^{\text {fit }}$ \\
\hline$(1,2,1)$ & $1.1185(22)$ & $0.9321(80)$ & 0.0199 \\
$(1,5,2)$ & $1.5721(55)$ & $0.8586(140)$ & -0.0052 \\
$(1,6,2)$ & $1.6804(76)$ & $0.8446(190)$ & -0.0030 \\
$(1,7,2)$ & $1.7949(40)$ & $0.8514(64)$ & 0.0063 \\
$(2,3,2)$ & $1.4653(19)$ & $0.9028(33)$ & 0.0068 \\
$(2,4,2)$ & $1.5757(21)$ & $0.8896(32)$ & 0.0125 \\
$(2,5,2)$ & $1.6820(30)$ & $0.8708(51)$ & 0.0152 \\
$(3,3,2)$ & $1.5732(27)$ & $0.8879(45)$ & 0.0144 \\
$(3,4,2)$ & $1.6793(33)$ & $0.8689(52)$ & 0.0203 \\
$(3,4,3)$ & $1.7660(36)$ & $0.8592(54)$ & -0.0035 \\
$(3,5,3)$ & $1.8677(43)$ & $0.8377(67)$ & -0.0002 \\
$(4,4,3)$ & $1.8692(48)$ & $0.8449(77)$ & 0.0056 \\
\hline \hline
\end{tabular}


Table 8: A part of lattice QCD results for the $3 \mathrm{Q}$ potential $V_{3 \mathrm{Q}}^{\text {latt }}$ at $\beta=6.0 .(i, j, k)$ denotes the $3 \mathrm{Q}$ system where the three quarks are put on $(i, 0,0)$, $(0, j, 0)$ and $(0,0, k)$ in $\mathbf{R}^{3}$ in the lattice unit. For each $3 \mathrm{Q}$ configuration, $V_{3 \mathrm{Q}}^{\text {latt }}$ is measured from the single-exponential fit as $\left\langle W_{3 \mathrm{Q}}\right\rangle=\bar{C} e^{-V_{3 \mathrm{Q}} T}$. The prefactor $\bar{C}$ physically means the magnitude of the ground-state component. The difference from the best-fit function $V_{3 \mathrm{Q}}^{\mathrm{fit}}$ in the $\mathrm{Y}$-ansatz is added. The listed values are measured in the lattice unit.

\begin{tabular}{lccr}
\hline \hline$(i, j, k)$ & $V_{3 \mathrm{Q}}^{\text {latt }}$ & $\bar{C}$ & $V_{3 \mathrm{Q}}^{\text {latt }}-V_{3 \mathrm{Q}}^{\text {fit }}$ \\
\hline$(0,1,1)$ & $0.6778(6)$ & $0.9784(24)$ & -0.0012 \\
$(0,1,2)$ & $0.8234(11)$ & $0.9712(45)$ & -0.0042 \\
$(0,1,3)$ & $0.9183(17)$ & $0.9769(65)$ & 0.0045 \\
$(0,1,4)$ & $0.9859(24)$ & $0.9589(92)$ & 0.0050 \\
$(0,1,5)$ & $1.0463(30)$ & $0.9495(112)$ & 0.0064 \\
$(0,1,6)$ & $1.1069(40)$ & $0.9595(152)$ & 0.0122 \\
$(0,1,7)$ & $1.1572(50)$ & $0.9374(192)$ & 0.0102 \\
$(0,2,2)$ & $0.9430(21)$ & $0.9586(78)$ & -0.0095 \\
$(0,2,3)$ & $1.0259(24)$ & $0.9607(91)$ & -0.0045 \\
$(0,2,4)$ & $1.0946(32)$ & $0.9657(120)$ & 0.0003 \\
$(0,2,5)$ & $1.1454(41)$ & $0.9282(149)$ & -0.0064 \\
$(0,2,6)$ & $1.2075(28)$ & $0.9464(76)$ & 0.0018 \\
$(0,2,7)$ & $1.2563(33)$ & $0.9262(90)$ & -0.0012 \\
$(0,3,3)$ & $1.0999(23)$ & $0.9566(62)$ & -0.0031 \\
$(0,3,4)$ & $1.1595(25)$ & $0.9454(67)$ & -0.0044 \\
$(0,3,5)$ & $1.2170(25)$ & $0.9426(65)$ & -0.0026 \\
$(0,3,6)$ & $1.2699(32)$ & $0.9327(90)$ & -0.0027 \\
$(0,3,7)$ & $1.3216(40)$ & $0.9232(110)$ & -0.0021 \\
$(0,3,8)$ & $1.3765(37)$ & $0.9241(92)$ & 0.0029 \\
$(0,4,4)$ & $1.2177(32)$ & $0.9394(87)$ & -0.0050 \\
$(0,4,5)$ & $1.2723(34)$ & $0.9336(96)$ & -0.0047 \\
$(0,4,6)$ & $1.3302(40)$ & $0.9418(110)$ & 0.0013 \\
$(0,4,7)$ & $1.3744(42)$ & $0.9128(108)$ & -0.0048 \\
$(0,4,8)$ & $1.4233(51)$ & $0.8982(138)$ & -0.0054 \\
$(0,5,5)$ & $1.3251(40)$ & $0.9265(112)$ & -0.0050 \\
$(0,5,6)$ & $1.3762(39)$ & $0.9187(108)$ & -0.0049 \\
$(0,5,7)$ & $1.4273(49)$ & $0.9110(131)$ & -0.0035 \\
$(0,5,8)$ & $1.4799(51)$ & $0.9079(140)$ & 0.0002 \\
$(0,6,6)$ & $1.4248(52)$ & $0.9047(136)$ & -0.0066 \\
$(0,6,7)$ & $1.4785(51)$ & $0.9062(130)$ & -0.0020 \\
$(0,6,8)$ & $1.5300(56)$ & $0.9020(146)$ & 0.0011 \\
$(0,7,7)$ & $1.5314(35)$ & $0.9058(59)$ & 0.0023 \\
$(0,7,8)$ & $1.5811(41)$ & $0.8971(66)$ & 0.0039 \\
$(0,8,8)$ & $1.6325(47)$ & $0.8924(76)$ & 0.0078 \\
$(1,1,1)$ & $0.7900(21)$ & $0.9588(98)$ & 0.0073 \\
$(1,1,2)$ & $0.8992(25)$ & $0.9707(118)$ & 0.0044 \\
$(1,1,3)$ & $0.9800(38)$ & $0.9578(182)$ & 0.0052 \\
$(1,1,4)$ & $1.0515(25)$ & $0.9677(99)$ & 0.0115 \\
$(1,1,5)$ & $1.1105(36)$ & $0.9578(135)$ & 0.0123 \\
$(1,1,6)$ & $1.1645(47)$ & $0.9449(175)$ & 0.0120 \\
\hline
\end{tabular}

Table 9: A part of lattice QCD results for the $3 \mathrm{Q}$ potential $V_{3 \mathrm{Q}}^{\text {latt }}$ at $\beta=6.0$. The notations are the same in Table 8 .

\begin{tabular}{|c|c|c|c|}
\hline$\overline{(\overline{(i, j, k)}}$ & $\overline{V_{3 \mathrm{Q}}^{\text {latt }}}$ & $\overline{\bar{C}}$ & $V_{3 \mathrm{Q}}^{\text {latt }}-V_{3 \mathrm{Q}}^{\mathrm{fit}}$ \\
\hline$(1,2,4)$ & $1.1185(31)$ & $0.9689(119)$ & 0.0043 \\
\hline$(1,2,5)$ & $1.1720(39)$ & $0.9458(142)$ & 0.0013 \\
\hline$(1,2,6)$ & $1.2283(49)$ & $0.9467(182)$ & 0.0043 \\
\hline$(1,2,7)$ & $1.2712(60)$ & $0.9015(208)$ & -0.0042 \\
\hline$(1,2,8)$ & $1.3314(66)$ & $0.9262(243)$ & 0.0058 \\
\hline$(1,3,3)$ & $1.1166(34)$ & $0.9582(132)$ & -0.0013 \\
\hline$(1,3,4)$ & $1.1783(35)$ & $0.9659(137)$ & 0.0018 \\
\hline$(1,3,5)$ & $1.2299(45)$ & $0.9453(174)$ & -0.0011 \\
\hline$(1,3,6)$ & $1.2877(32)$ & $0.9513(87)$ & 0.0045 \\
\hline$(1,3,7)$ & $1.3293(58)$ & $0.9095(210)$ & -0.0047 \\
\hline$(1,3,8)$ & $1.3863(40)$ & $0.9214(103)$ & 0.0027 \\
\hline$(1,4,4)$ & $1.2296(29)$ & $0.9442(75)$ & -0.0030 \\
\hline$(1,4,5)$ & $1.2863(29)$ & $0.9484(78)$ & 0.0006 \\
\hline$(1,4,6)$ & $1.3326(35)$ & $0.9236(90)$ & -0.0043 \\
\hline$(1,4,7)$ & $1.3864(40)$ & $0.9249(102)$ & -0.0004 \\
\hline$(1,4,8)$ & $1.4401(44)$ & $0.9249(117)$ & 0.0042 \\
\hline$(1,5,5)$ & $1.3363(37)$ & $0.9370(99)$ & -0.0013 \\
\hline$(1,5,6)$ & $1.3879(37)$ & $0.9316(99)$ & 0.0001 \\
\hline$(1,5,7)$ & $1.4318(44)$ & $0.9039(116)$ & -0.0053 \\
\hline$(1,5,8)$ & $1.4837(47)$ & $0.9001(127)$ & -0.0020 \\
\hline$(1,6,6)$ & $1.4344(52)$ & $0.9115(138)$ & -0.0029 \\
\hline$(1,6,7)$ & $1.4870(55)$ & $0.9115(150)$ & 0.0009 \\
\hline$(1,6,8)$ & $1.5370(53)$ & $0.9037(142)$ & 0.0030 \\
\hline$(1,7,7)$ & $1.5258(65)$ & $0.8729(165)$ & -0.0084 \\
\hline$(1,7,8)$ & $1.5743(69)$ & $0.8624(168)$ & -0.0075 \\
\hline$(1,8,8)$ & $1.6370(44)$ & $0.8888(69)$ & 0.0080 \\
\hline$(2,2,2)$ & $1.0405(34)$ & $0.9669(132)$ & 0.0004 \\
\hline$(2,2,3)$ & $1.0963(31)$ & $0.9462(115)$ & -0.0045 \\
\hline$(2,2,4)$ & $1.1579(37)$ & $0.9570(144)$ & -0.0002 \\
\hline$(2,2,5)$ & $1.2108(49)$ & 0.9396(181) & -0.0016 \\
\hline$(2,2,6)$ & $1.2676(32)$ & $0.9446(90)$ & 0.0031 \\
\hline$(2,2,7)$ & $1.3035(69)$ & $0.8803(246)$ & -0.0117 \\
\hline$(2,2,8)$ & $1.3613(42)$ & $0.9000(112)$ & -0.0035 \\
\hline$(2,3,3)$ & $1.1461(39)$ & $0.9322(147)$ & -0.0087 \\
\hline$(2,3,4)$ & $1.1994(40)$ & $0.9247(149)$ & -0.0089 \\
\hline$(2,3,5)$ & $1.2525(52)$ & $0.9200(188)$ & -0.0079 \\
\hline$(2,3,6)$ & $1.3114(32)$ & $0.9368(85)$ & 0.0002 \\
\hline$(2,3,7)$ & $1.3499(71)$ & $0.8852(256)$ & -0.0110 \\
\hline$(2,3,8)$ & $1.4000(79)$ & $0.8775(280)$ & -0.0099 \\
\hline$(2,4,4)$ & $1.2565(28)$ & $0.9443(80)$ & -0.0027 \\
\hline$(2,4,5)$ & $1.3041(34)$ & $0.9274(89)$ & -0.0053 \\
\hline$(2,4,6)$ & $1.3549(36)$ & $0.9207(100)$ & -0.0041 \\
\hline$(2,4,7)$ & $1.4006(37)$ & $0.8990(98)$ & -0.0072 \\
\hline$(2,4,8)$ & $1.4535(49)$ & $0.8980(133)$ & -0.0026 \\
\hline$(2,5,5)$ & $1.3505(42)$ & $0.9129(111)$ & -0.0077 \\
\hline$(2,5,6)$ & $1.4074(42)$ & $0.9272(116)$ & 0.0007 \\
\hline$(2,5,7)$ & $1.4520(48)$ & $0.9040(126)$ & -0.0028 \\
\hline$(2,6,6)$ & $1.4515(49)$ & $0.9055(130)$ & -0.0029 \\
\hline$(2,6,7)$ & $1.4920(53)$ & $0.8722(135)$ & -0.0098 \\
\hline
\end{tabular}


Table 10: A part of lattice QCD results for the $3 \mathrm{Q}$ potential $V_{3 \mathrm{Q}}^{\text {latt }}$ at $\beta=6.0$. The notations are the same in Table 8 .

\begin{tabular}{|c|c|c|c|}
\hline$\overline{(\overline{(i, j, k)}}$ & $\bar{V}_{3 \mathrm{Q}}^{\text {latt }}$ & $\overline{\bar{C}}$ & $=V_{3 \mathrm{Q}}^{\text {latt }}-V_{3 \mathrm{Q}}^{\text {fit }}$ \\
\hline$(2,6,8)$ & $1.5528(55)$ & $0.8950(133)$ & 0.0039 \\
\hline$(2,7,7)$ & $1.5442(61)$ & $0.8727(163)$ & -0.0044 \\
\hline$(2,7,8)$ & $1.5910(71)$ & $0.8585(177)$ & -0.0042 \\
\hline$(2,8,8)$ & $1.6411(84)$ & $0.8518(213)$ & -0.0004 \\
\hline$(3,3,3)$ & $1.1951(31)$ & $0.9424(86)$ & -0.0060 \\
\hline$(3,3,4)$ & $1.2421(28)$ & $0.9308(77)$ & -0.0080 \\
\hline$(3,3,5)$ & $1.2936(34)$ & $0.9280(93)$ & -0.0059 \\
\hline$(3,3,6)$ & $1.3404(42)$ & $0.9075(111)$ & -0.0082 \\
\hline$(3,3,8)$ & $1.4434(52)$ & $0.8992(141)$ & -0.0020 \\
\hline$(3,4,4)$ & $1.2894(32)$ & $0.9292(79)$ & -0.0066 \\
\hline$(3,4,5)$ & $1.3350(34)$ & $0.9142(88)$ & -0.0081 \\
\hline$(3,4,6)$ & $1.3825(40)$ & $0.9014(103)$ & -0.0082 \\
\hline$(3,4,7)$ & $1.4353(49)$ & $0.9033(131)$ & -0.0029 \\
\hline$(3,4,8)$ & $1.4867(56)$ & $0.8987(145)$ & 0.0011 \\
\hline$(3,5,6)$ & $1.4273(42)$ & $0.8929(114)$ & -0.0075 \\
\hline$(3,5,7)$ & $1.4791(53)$ & $0.8931(138)$ & -0.0023 \\
\hline$(3,5,8)$ & $1.5260(50)$ & $0.8770(129)$ & -0.0020 \\
\hline$(3,6,6)$ & $1.4737(61)$ & $0.8812(162)$ & -0.0064 \\
\hline$(3,6,7)$ & $1.5234(51)$ & $0.8769(124)$ & -0.0024 \\
\hline$(3,6,8)$ & $1.5734(61)$ & $0.8709(147)$ & 0.0015 \\
\hline$(3,7,7)$ & $1.5655(77)$ & $0.8535(203)$ & -0.0054 \\
\hline$(3,7,8)$ & $1.6177(77)$ & $0.8550(189)$ & 0.0013 \\
\hline$(3,8,8)$ & $1.6666(92)$ & $0.8458(222)$ & 0.0054 \\
\hline$(4,4,4)$ & $1.3255(46)$ & $0.9047(119)$ & -0.0126 \\
\hline$(4,4,5)$ & $1.3765(41)$ & $0.9116(111)$ & -0.0061 \\
\hline$(4,4,6)$ & $1.4262(49)$ & $0.9088(129)$ & -0.0021 \\
\hline$(4,4,7)$ & $1.4745(60)$ & $0.8985(155)$ & 0.0000 \\
\hline$(4,4,8)$ & $1.5243(69)$ & $0.8925(174)$ & 0.0034 \\
\hline$(4,5,5)$ & $1.4165(49)$ & $0.8903(132)$ & -0.0086 \\
\hline$(4,5,6)$ & $1.4628(51)$ & $0.8808(124)$ & -0.0065 \\
\hline$(4,5,7)$ & $1.5155(59)$ & $0.8868(155)$ & 0.0012 \\
\hline$(4,5,8)$ & $1.5651(63)$ & $0.8786(162)$ & 0.0053 \\
\hline$(4,6,6)$ & $1.5198(65)$ & $0.9031(175)$ & 0.0076 \\
\hline$(4,6,7)$ & $1.5549(66)$ & $0.8608(163)$ & -0.0013 \\
\hline$(4,6,8)$ & $1.6045(75)$ & $0.8552(188)$ & 0.0036 \\
\hline$(5,5,5)$ & $1.4653(75)$ & $0.8984(203)$ & 0.0000 \\
\hline$(5,5,6)$ & $1.5074(63)$ & $0.8814(158)$ & -0.0003 \\
\hline$(5,5,7)$ & $1.5452(80)$ & $0.8475(199)$ & -0.0061 \\
\hline$(5,5,8)$ & $1.6008(83)$ & $0.8574(208)$ & 0.0050 \\
\hline$(5,6,6)$ & $1.5511(72)$ & $0.8689(181)$ & 0.0026 \\
\hline$(5,6,8)$ & $1.6385(81)$ & $0.8343(195)$ & 0.0040 \\
\hline$(5,7,7)$ & $1.6354(89)$ & $0.8288(216)$ & 0.0029 \\
\hline$(5,7,8)$ & $1.6878(93)$ & $0.8325(219)$ & 0.0127 \\
\hline$(6,6,6)$ & $1.5953(108)$ & $0.8605(272)$ & 0.0076 \\
\hline$(6,6,7)$ & $1.6328(91)$ & $0.8285(222)$ & 0.0040 \\
\hline$(6,6,8)$ & $1.6956(46)$ & $0.8595(67)$ & 0.0244 \\
\hline$(6,8,8)$ & $1.7701(138)$ & $0.7972(325)$ & 0.0193 \\
\hline$(7,7,8)$ & $1.7666(139)$ & $0.7915(326)$ & 0.0188 \\
\hline$(7,8,8)$ & $1.8114(166)$ & $0.7795(376)$ & 0.0243 \\
\hline
\end{tabular}

Table 11: A part of lattice QCD results for the $3 \mathrm{Q}$ potential $V_{3 \mathrm{Q}}^{\text {latt }}$ at $\beta=6.0 .(l, m, n)$ denotes the $3 \mathrm{Q}$ system where the three quarks are put on $(l, 0,0),(-m, 0,0)$ and $(0, n, 0)$ in $\mathbf{R}^{3}$ in the lattice unit. The other notations are the same in Table 8 .

\begin{tabular}{cccc}
\hline \hline$(l, m, n)$ & $V_{3 \mathrm{Q}}^{\text {latt }}$ & $\bar{C}$ & $V_{3 \mathrm{Q}}^{\text {latt }}-V_{3 \mathrm{Q}}^{\text {fit }}$ \\
\hline$(1,2,1)$ & $0.9334(16)$ & $0.9726(64)$ & 0.0098 \\
$(1,5,2)$ & $1.2001(26)$ & $0.9529(74)$ & 0.0061 \\
$(1,6,2)$ & $1.2467(58)$ & $0.9211(214)$ & 0.0009 \\
$(2,3,2)$ & $1.1436(22)$ & $0.9600(61)$ & 0.0053 \\
$(2,4,2)$ & $1.2017(26)$ & $0.9555(72)$ & 0.0094 \\
$(2,5,2)$ & $1.2543(31)$ & $0.9418(84)$ & 0.0106 \\
$(2,6,2)$ & $1.3033(37)$ & $0.9218(105)$ & 0.0097 \\
$(2,6,3)$ & $1.3496(44)$ & $0.9202(120)$ & -0.0007 \\
$(3,3,2)$ & $1.2022(27)$ & $0.9537(77)$ & 0.0101 \\
$(3,4,2)$ & $1.2563(32)$ & $0.9405(90)$ & 0.0130 \\
$(3,4,3)$ & $1.2971(31)$ & $0.9330(86)$ & -0.0011 \\
$(3,5,3)$ & $1.3467(40)$ & $0.9215(108)$ & 0.0006 \\
$(4,4,3)$ & $1.3440(44)$ & $0.9147(117)$ & -0.0008 \\
\hline \hline
\end{tabular}




\begin{tabular}{lccccl}
\hline \hline & $\beta$ & $\sigma_{\Delta}$ & $A_{\Delta}$ & $C_{\Delta}$ & $\chi^{2} / N_{\mathrm{DF}}$ \\
\hline $3 \mathrm{Q}_{\Delta}$ & 5.7 & $0.0858(16)$ & $0.1410(64)$ & $0.9334(210)$ & 10.1 \\
$3 \mathrm{Q}_{\Delta}$ (Latt. Coul.) & & $0.0868(14)$ & $0.1296(51)$ & $0.9146(173)$ & 5.12 \\
\hline $3 \mathrm{Q}_{\Delta}$ & 5.8 & $0.0581(4)$ & $0.1197(19)$ & $0.8964(55)$ & 13.7 \\
$3 \mathrm{Q}_{\Delta}$ (Latt. Coul.) & & $0.0583(3)$ & $0.1110(18)$ & $0.8872(54)$ & 13.6 \\
\hline $3 \mathrm{Q}_{\Delta}$ & 6.0 & $0.0264(2)$ & $0.1334(11)$ & $0.9490(36)$ & 3.74 \\
$3 \mathrm{Q}_{\Delta}$ (Latt. Coul.) & & $0.0268(2)$ & $0.1227(10)$ & $0.9361(34)$ & 2.89 \\
\hline \hline
\end{tabular}

Table 12: The fit analysis of the lattice QCD data $V_{3 \mathrm{Q}}^{\text {latt }}$ with the $\Delta$-ansatz at each $\beta$. We list the best-fit parameter set $\left(\sigma_{\Delta}, A_{\Delta}, C_{\Delta}\right)$ in the function form as $-A_{\Delta} \sum_{i<j} \frac{1}{\left|\mathbf{r}_{i}-\mathbf{r}_{j}\right|}+\sigma_{\Delta} \sum_{i<j}\left|\mathbf{r}_{i}-\mathbf{r}_{j}\right|+C_{\Delta}$. The label of (Latt. Coul.) means the fit with the lattice Coulomb potential instead of the continuum Coulomb potential. The listed values are measured in the lattice unit.

\begin{tabular}{ccccccc}
\hline \hline$\beta$ & $\sigma_{\mathrm{GY}}$ & $A_{\mathrm{GY}}$ & $C_{\mathrm{GY}}$ & $R_{\text {core }}[a]$ & $R_{\text {core }}[\mathrm{fm}]$ & $\chi^{2} / N_{\mathrm{DF}}$ \\
\hline 5.8 & $0.1054(6)$ & $0.1354(18)$ & $0.9569(53)$ & 0.57 & 0.08 & 2.63 \\
6.0 & $0.0480(4)$ & $0.1451(11)$ & $0.9837(33)$ & 0.79 & 0.08 & 1.23 \\
\hline \hline
\end{tabular}

Table 13: The fit analysis of the lattice QCD data $V_{3 \mathrm{Q}}^{\text {latt }}$ with the generalized Y-ansatz at each $\beta$. We list the best-fit parameter set $\left(\sigma_{\mathrm{GY}}, A_{\mathrm{GY}}, C_{\mathrm{GY}}, R_{\mathrm{core}}\right)$ in the lattice unit at $\beta=5.8$ and 6.0. The flux-tube core radius $R_{\text {core }}$ in the physical unit is added. 


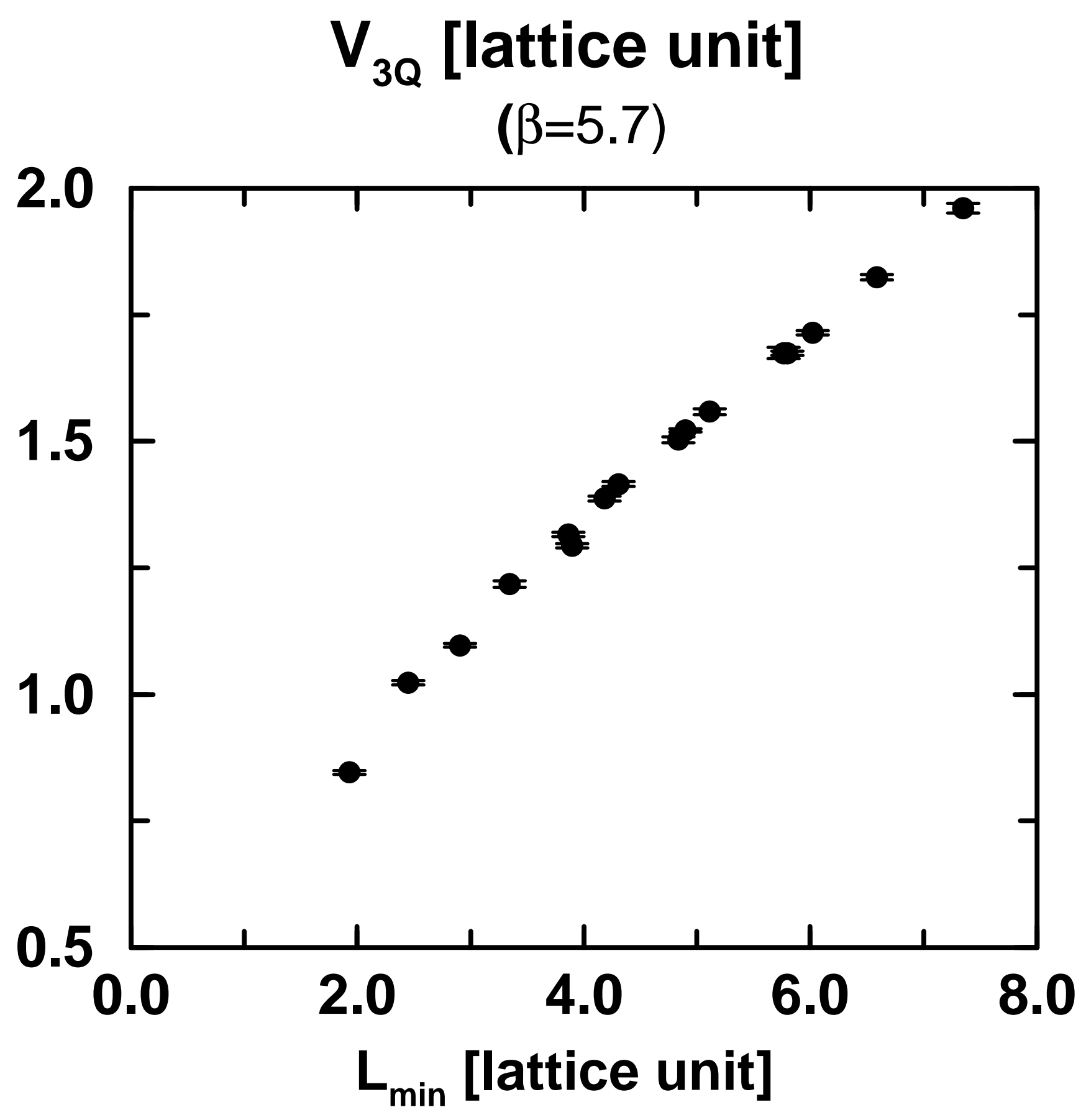




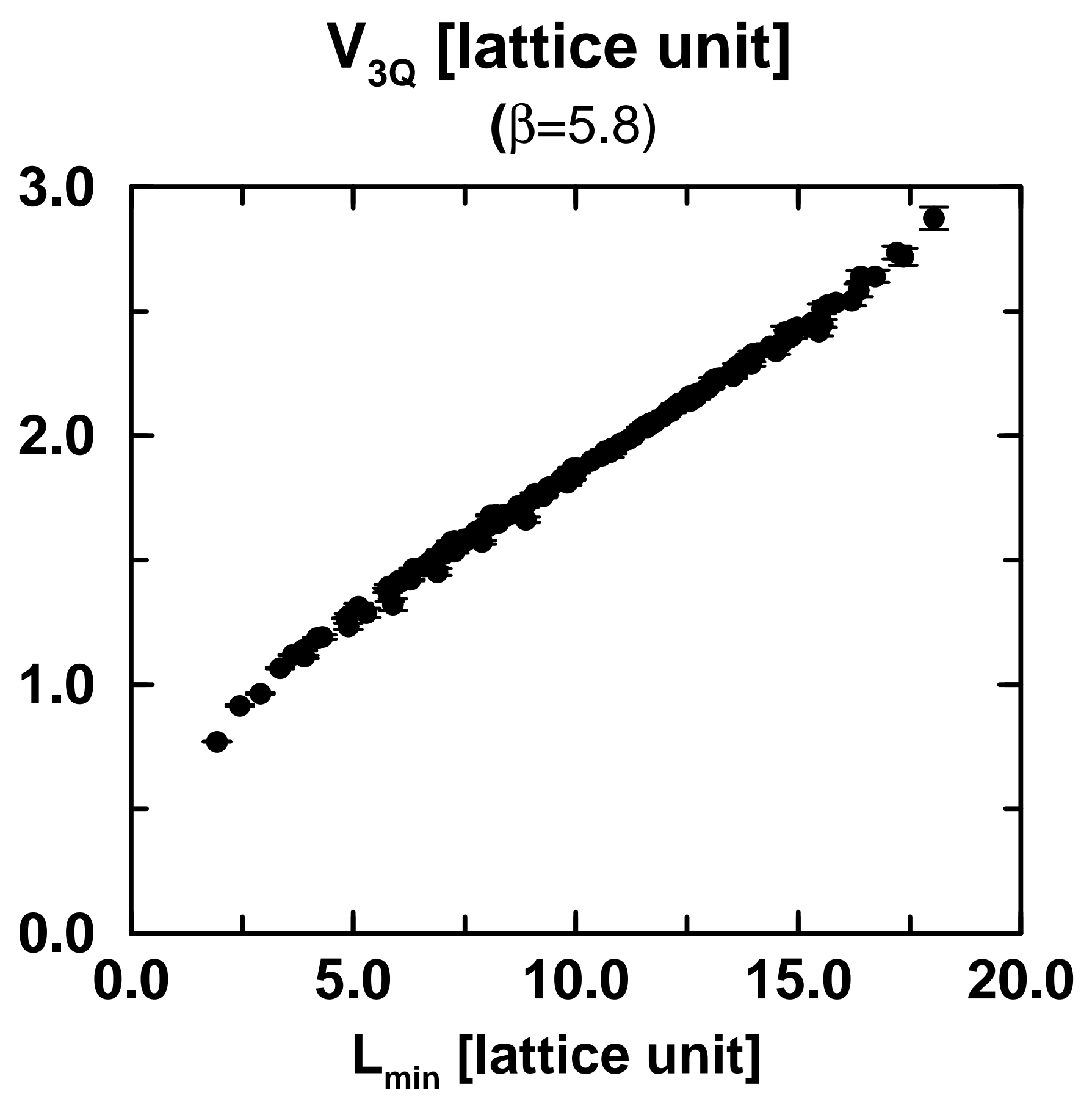




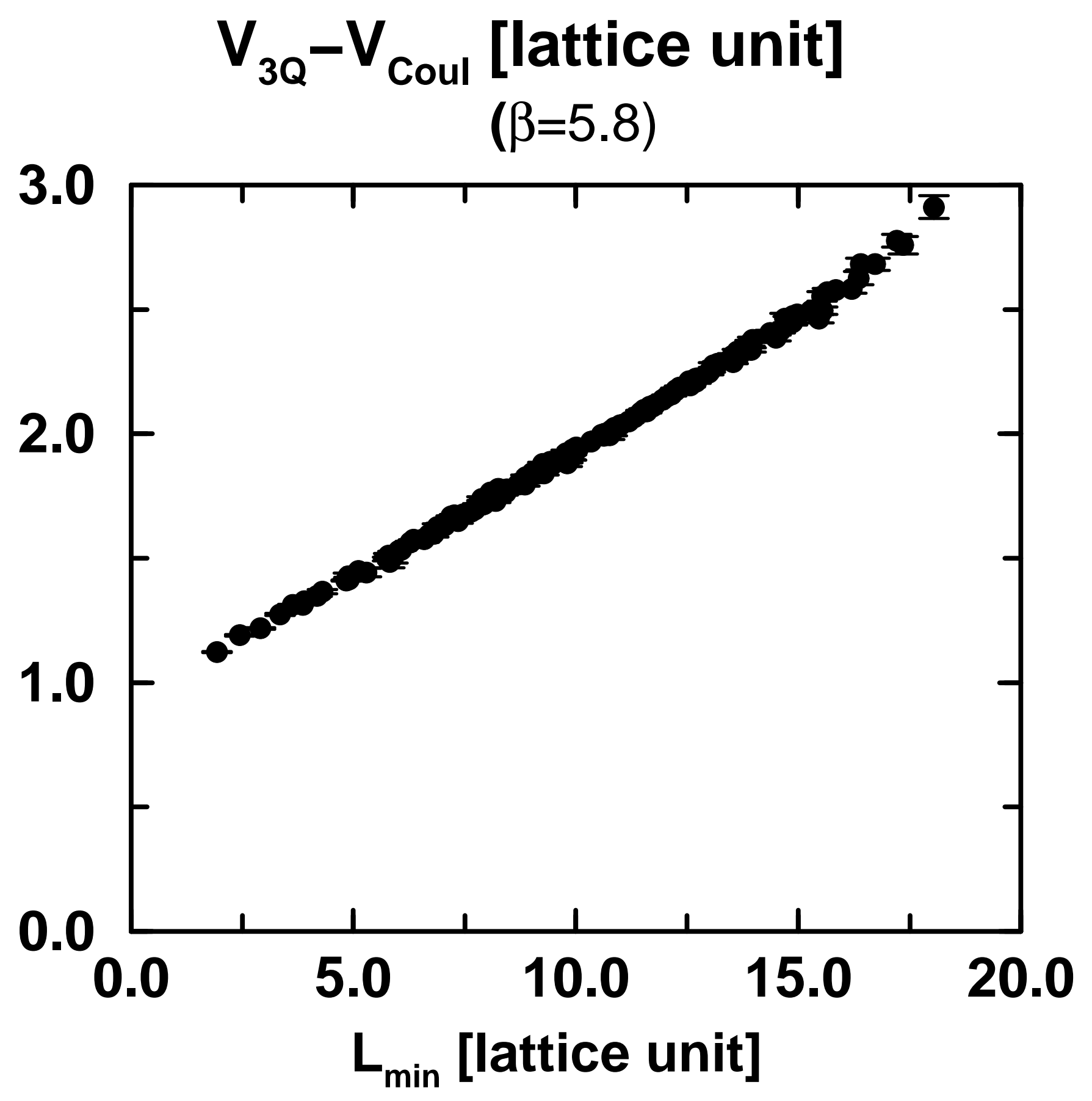




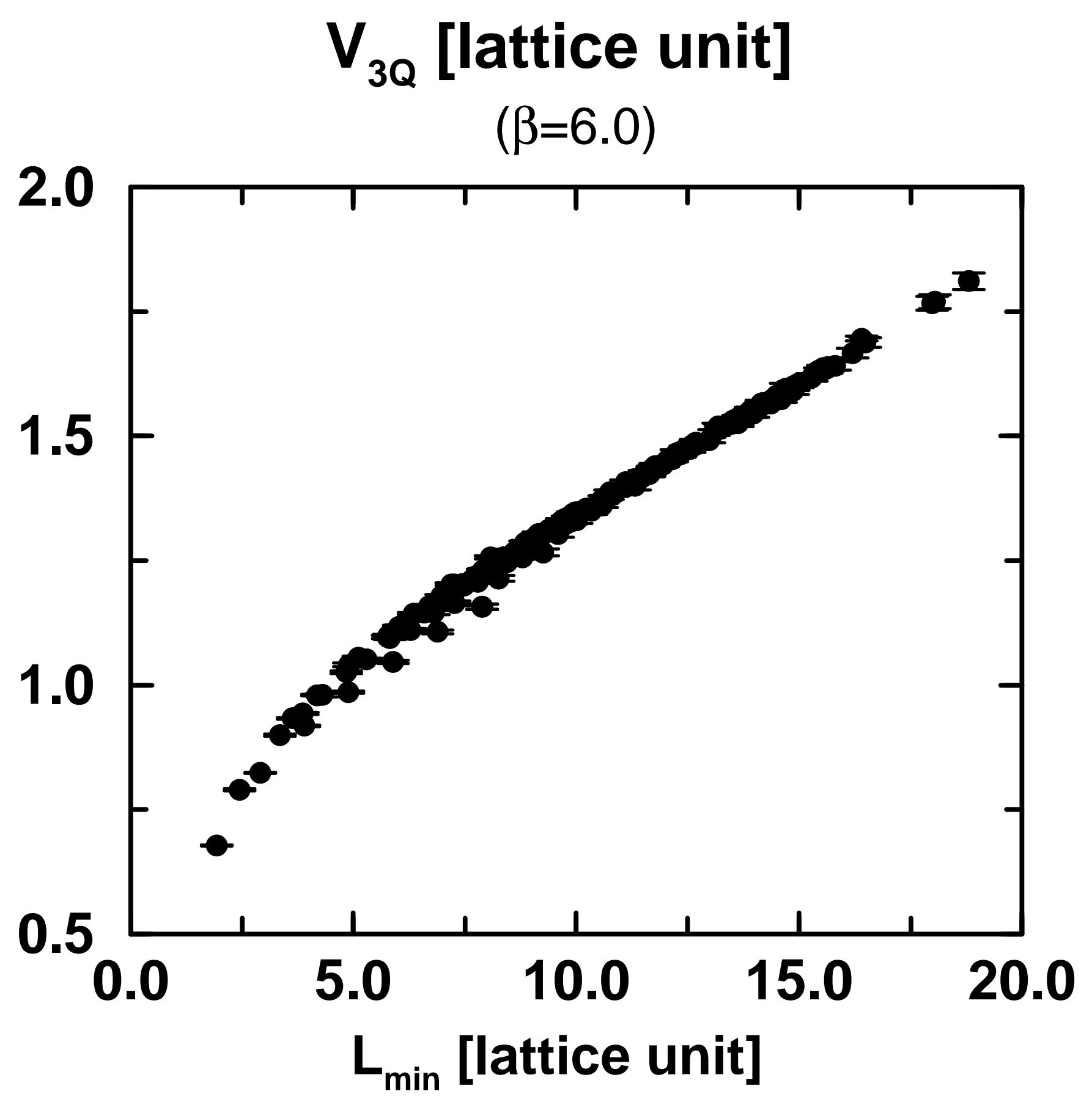




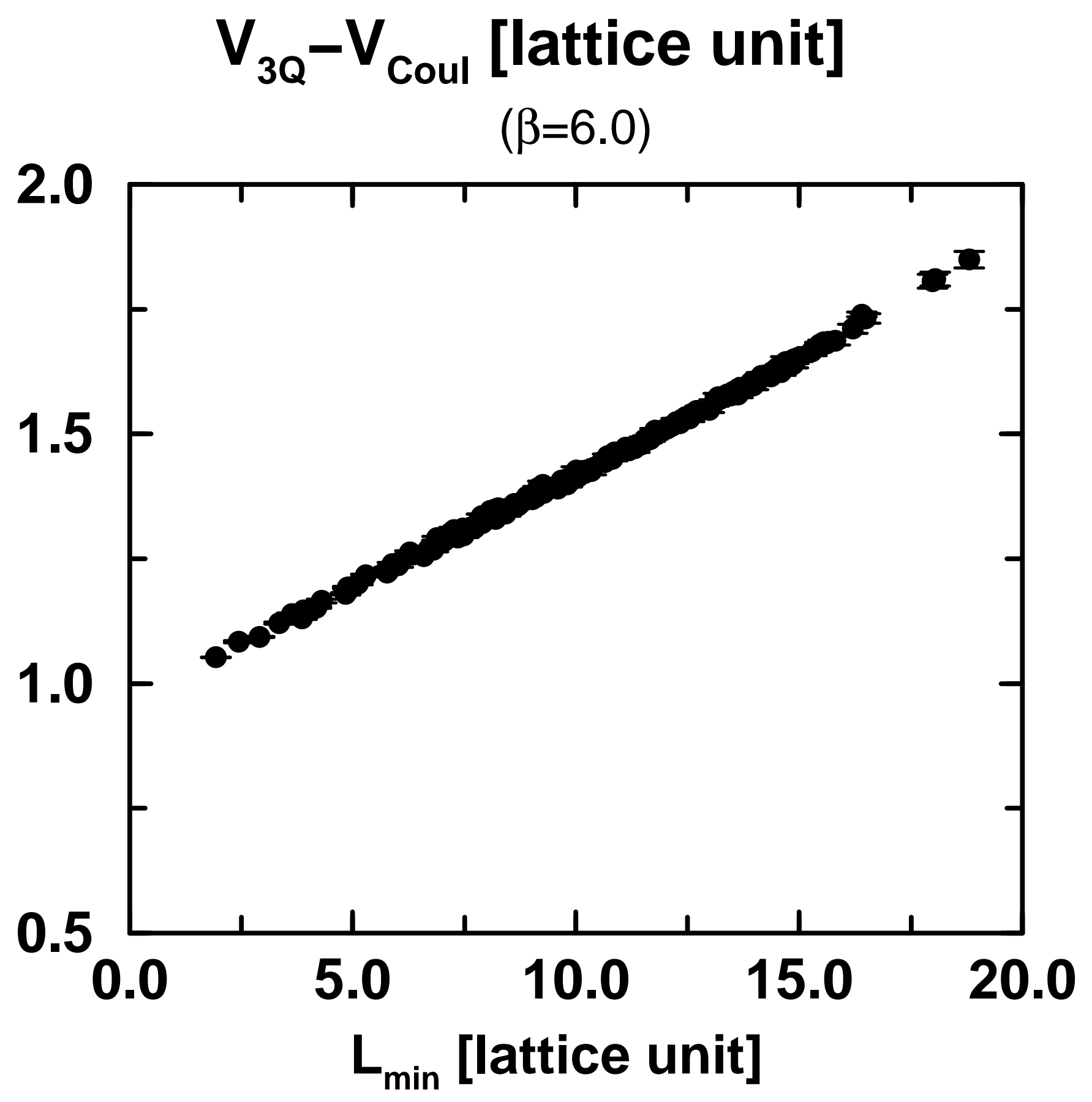



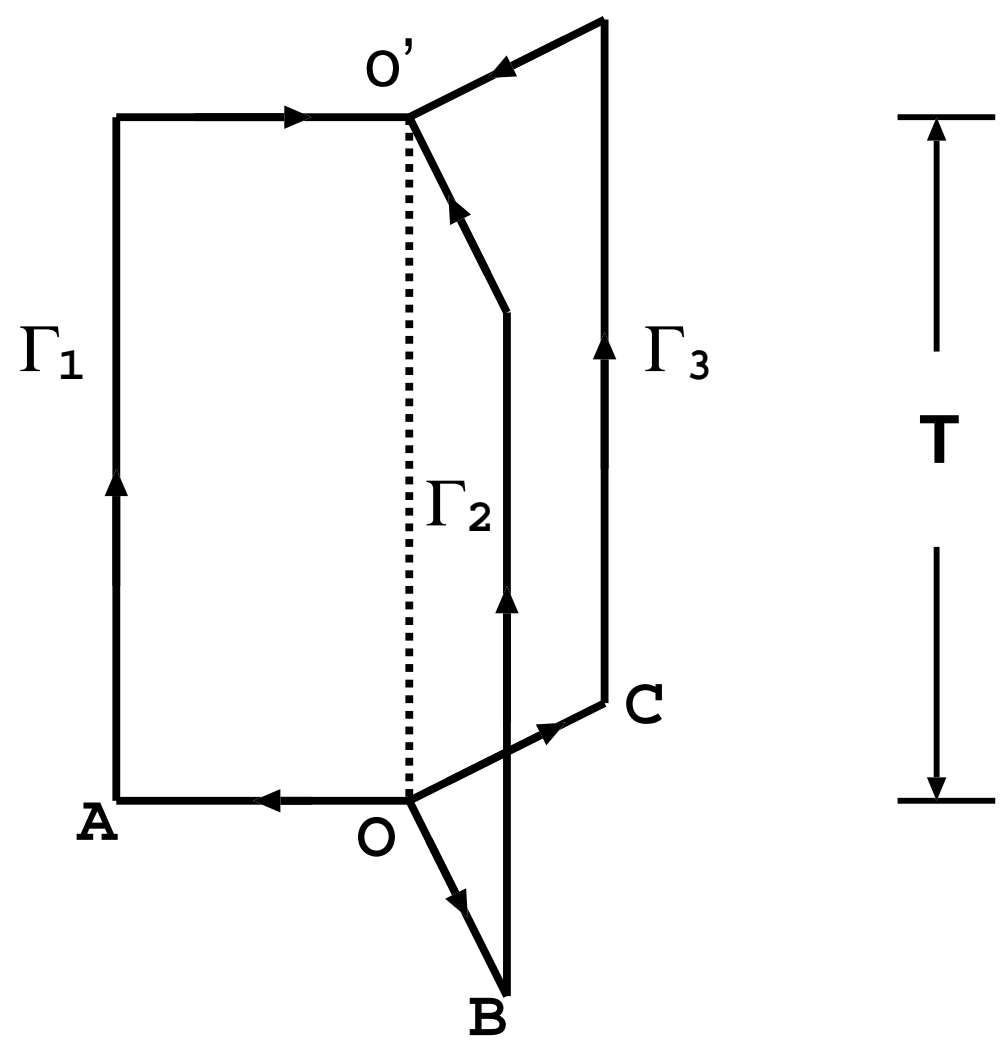


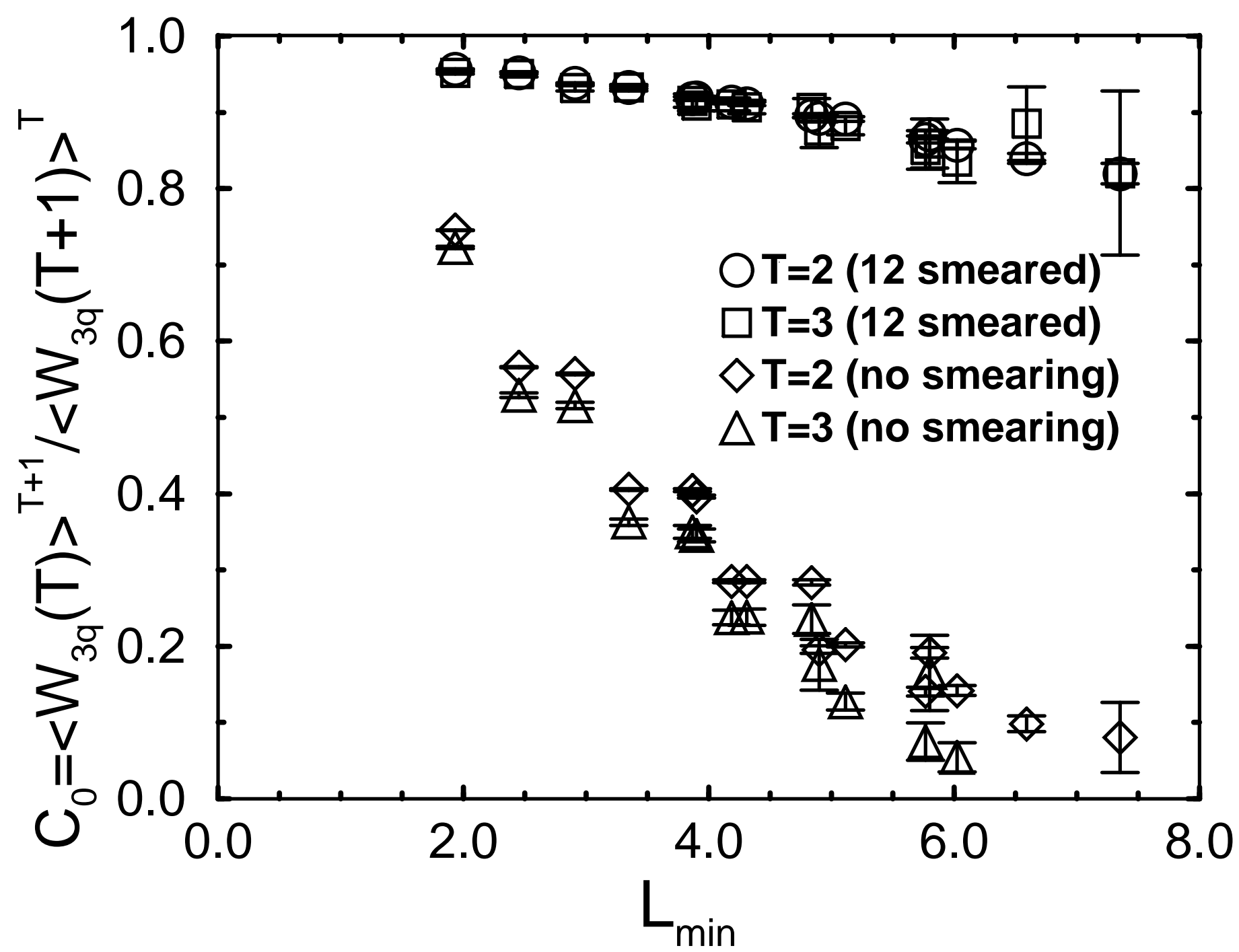




\section{$1 / r$ vs $V^{L C}$}

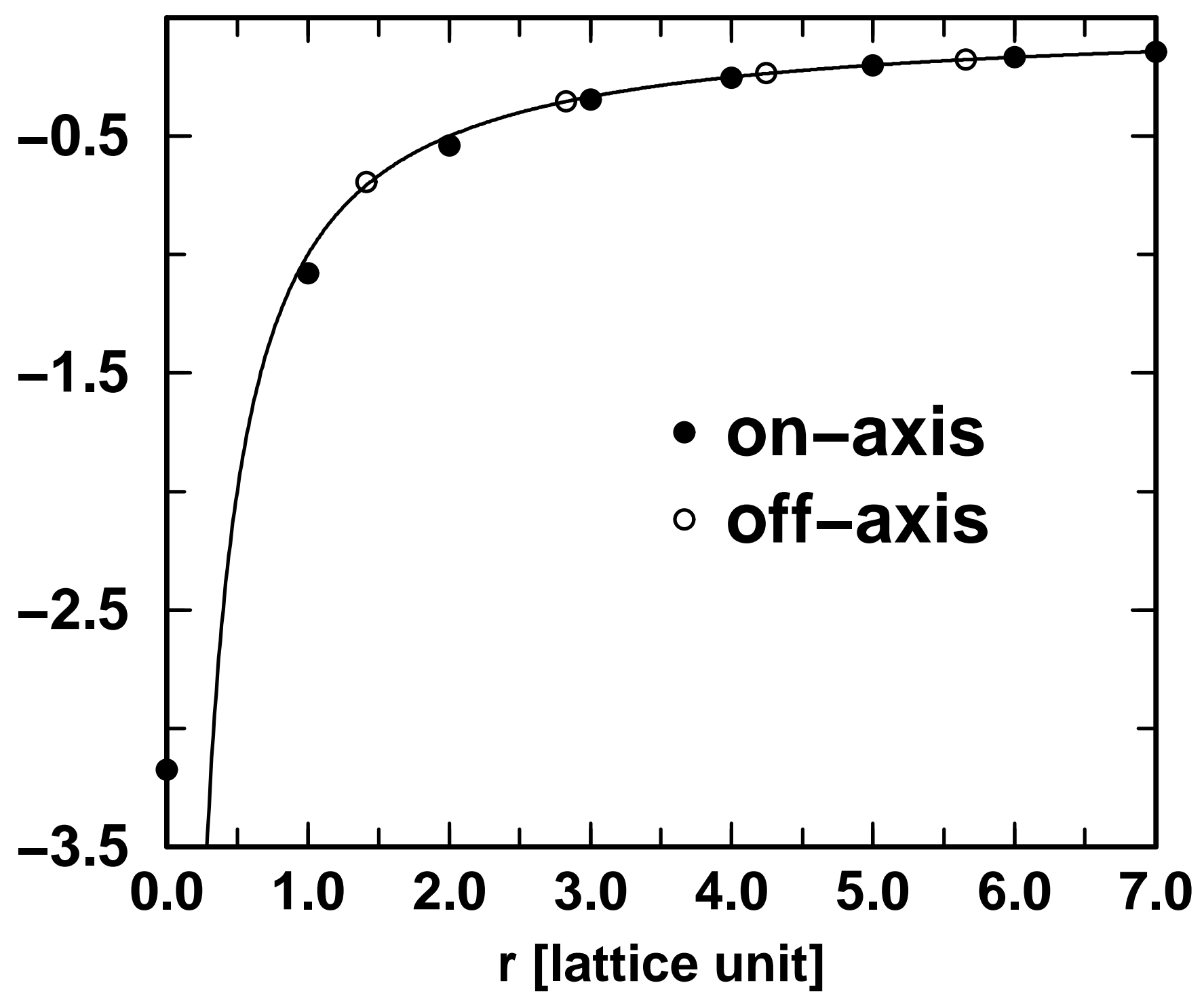




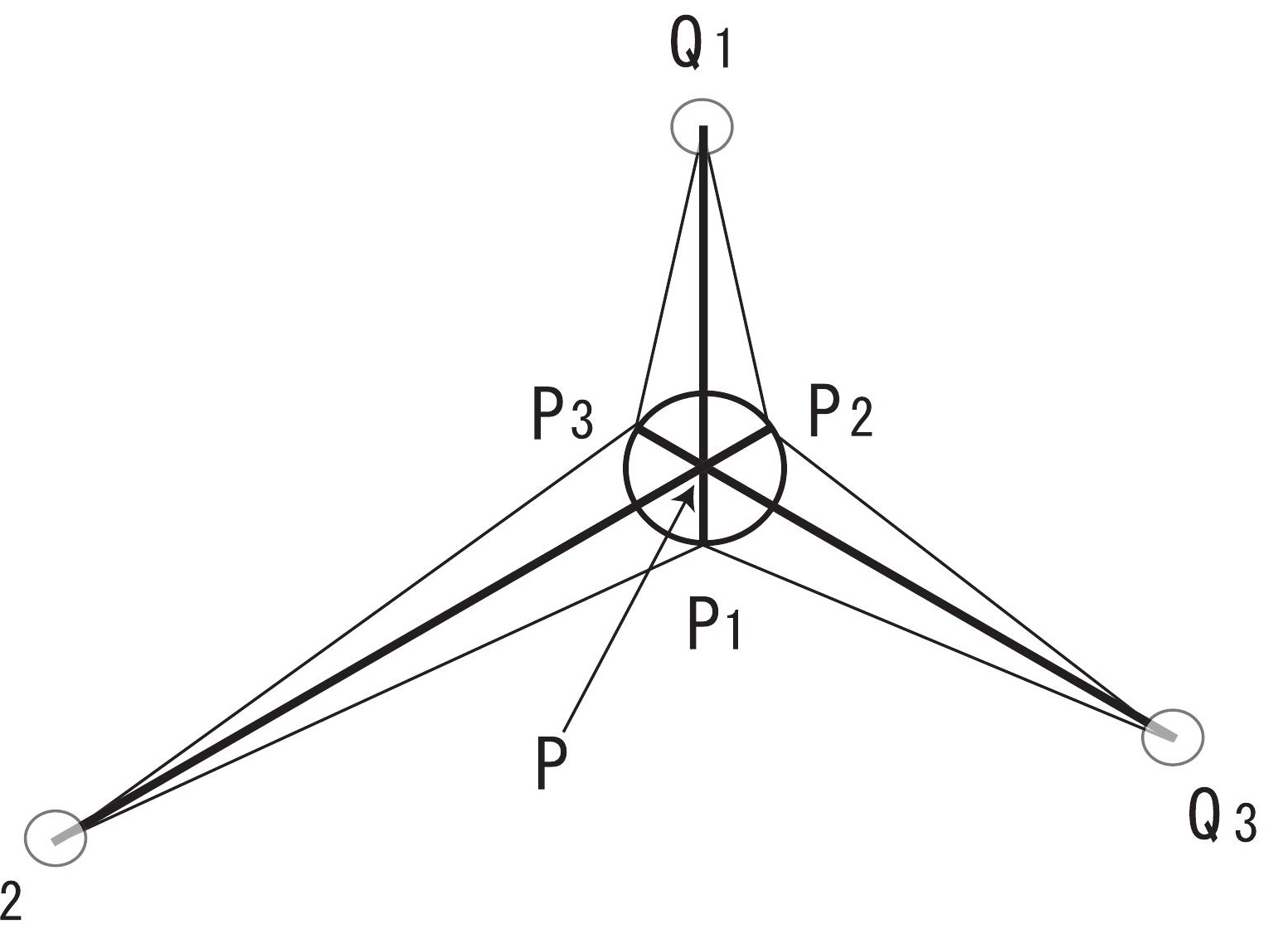




\section{quark-antiquark potential \\ $(\beta=6.0)$}

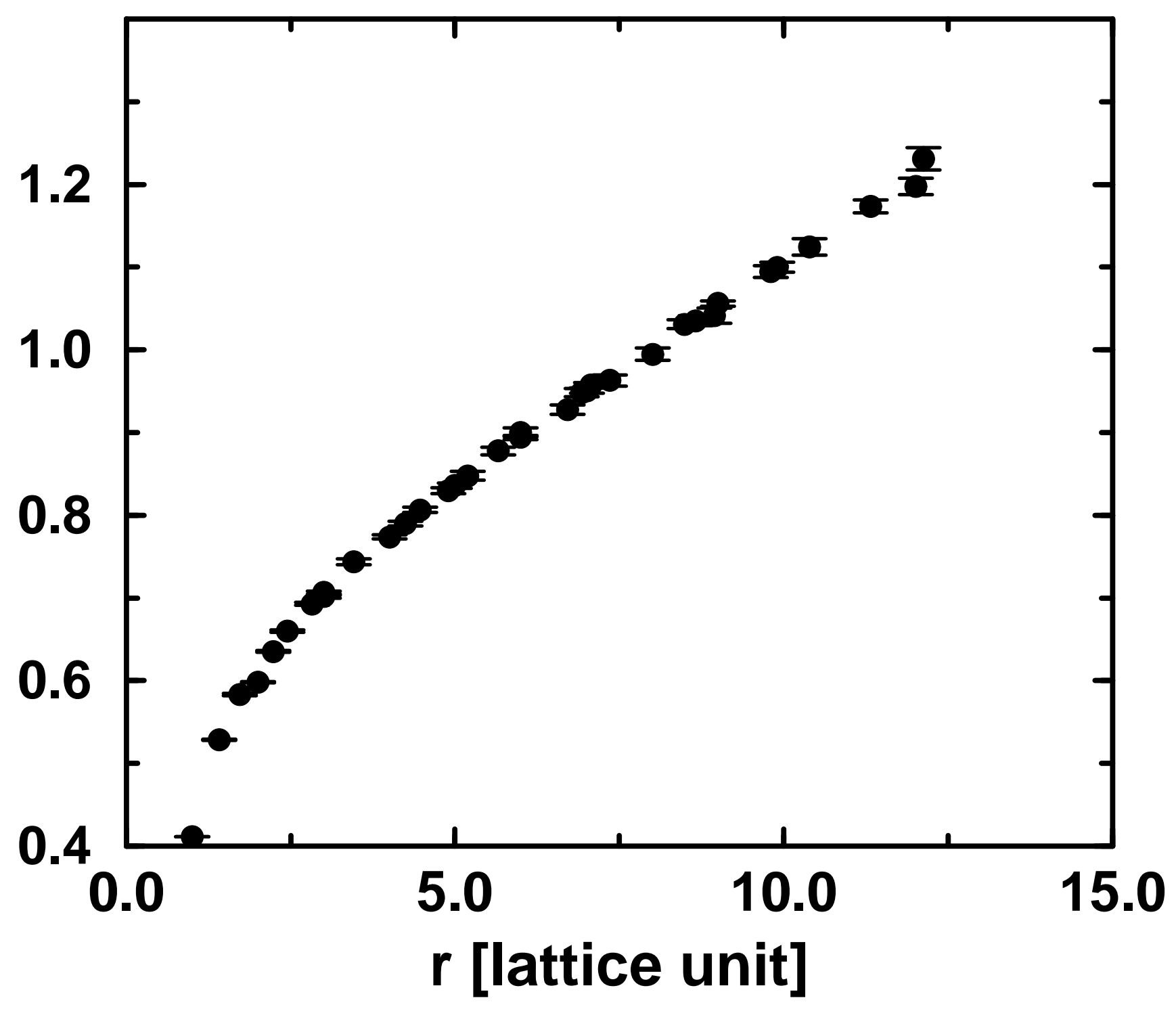




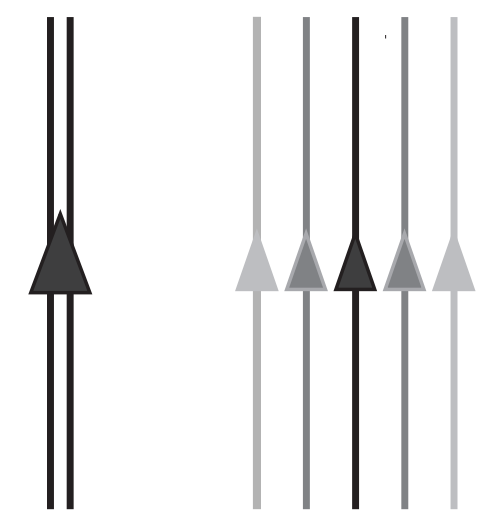

(a)

(b)

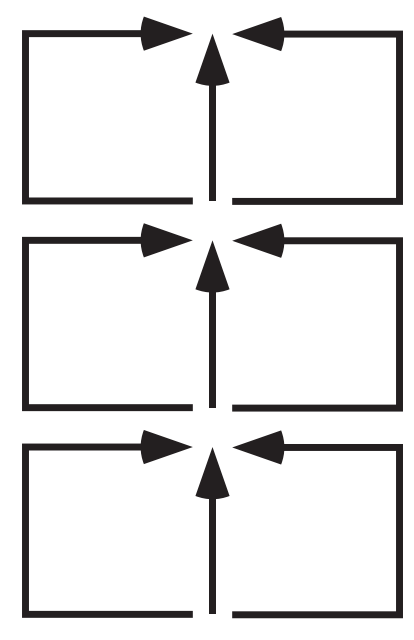

(c) $\varphi(r)$

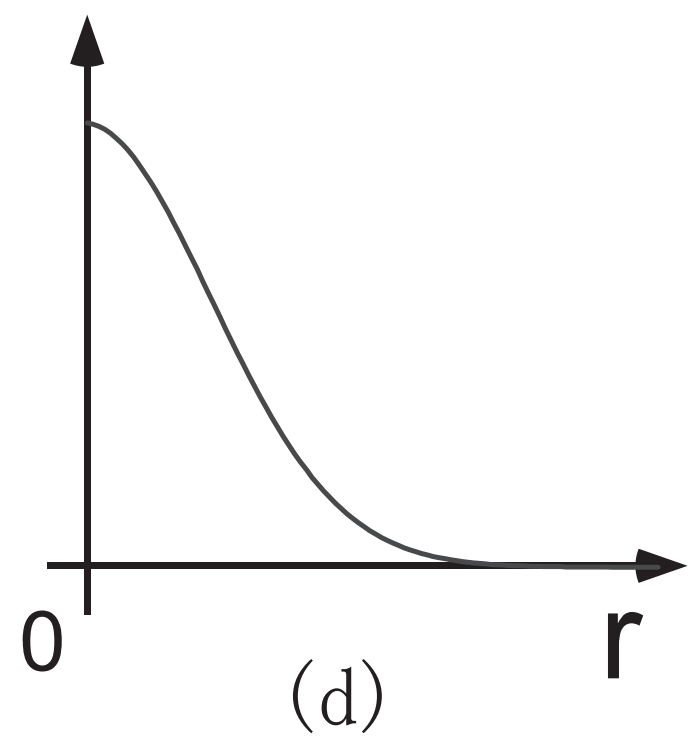




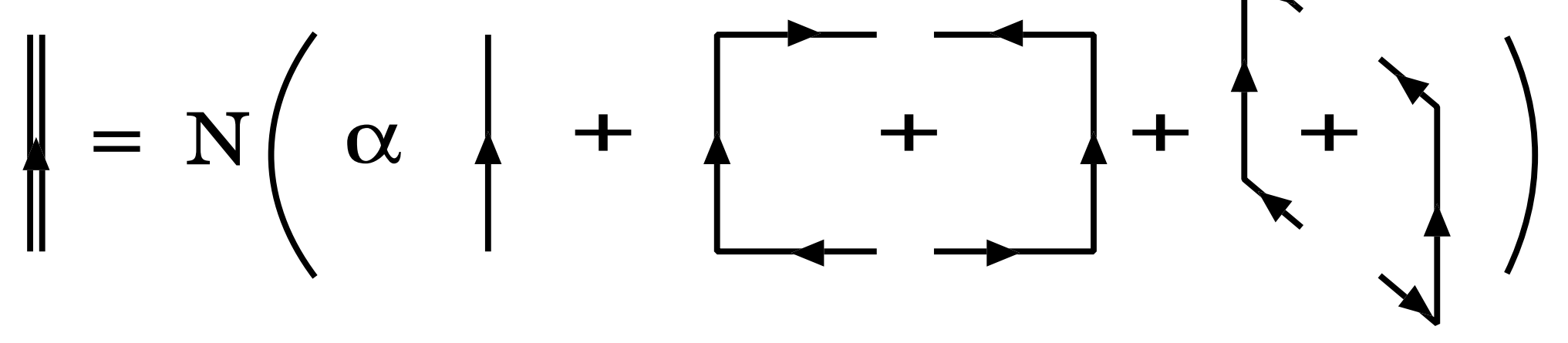

